\title{
NMR-guided design of potent and selective EphA4 agonistic ligands
}

\author{
Carlo Baggio, ${ }^{1}$ Anna Kulinich, ${ }^{1}$ Cassandra N. Dennys, ${ }^{2}$ Rochelle Rodrigo, ${ }^{2}$ Kathrin Meyer, ${ }^{2}$ Iryna \\ Ethell, ${ }^{1}$ and Maurizio Pellecchia ${ }^{1, *}$
}

1 Division of Biomedical Sciences, School of Medicine, University of California Riverside, 900

University Avenue, Riverside, CA 92521, USA.

2 Nationwide Children's Hospital, 700 Children's Drive, Columbus, $\mathrm{OH} 43205$, USA.

* Correspondence: Maurizio.Pellecchia@ucr.edu

\section{Content:}

Table S1. Compound ID, chemical structures and binding properties of EphA4 targeting agents (page S2).

Table S2. Data collection and Refinement statistics for 150D4 (page S4).

Table S3. In vivo PK/brain exposure studies (page S5).

Figure S1. HPLC traces, high resolution mass, and 1D ${ }^{1} \mathrm{H}$ NMR spectra for tested compounds in Table 6 (page S6).

Figure S2. General scheme for the synthesis of compounds 33(150D4) and 30 (page S11).

Figure S3. General scheme for the synthesis of compounds 31 and 30 (page S12).

Figure S4. General synthetic scheme, high-resolution mass, and 1D ${ }^{1} \mathrm{H}$ NMR spectra for compounds in Table 1 (page S13).

Figure S5. General synthetic scheme, high-resolution mass, and 1D ${ }^{1} \mathrm{H}$ NMR spectra for compounds in Table 2 (page S19).

Figure S6. General synthetic scheme, high-resolution mass, and 1D ${ }^{1} \mathrm{H}$ NMR spectra for compounds in Table 3 (page S22). 
Figure S7. General synthetic scheme, high-resolution mass, and 1D ${ }^{1} \mathrm{H}$ NMR spectra for compounds in Table 4 (page S27).

Figure S8. General synthetic scheme, high-resolution mass, and 1D ${ }^{1} \mathrm{H}$ NMR spectra for compounds in Table 5 (page S31).

Figure S9. WB data that generated the plots in Figure 5C,D (page S38).

Table S1. Compound ID, chemical structures and binding properties of EphA4 targeting agents. $\mathrm{K}_{\mathrm{d}}$ and thermodynamic binding parameters were obtained by isothermal titration calorimetry assays against recombinant EphA4 ligand binding domains. $\Delta \delta$ indicates chemical shift perturbations induced by test ligands (at $20 \mu \mathrm{M}$ concentration) to $1 \mathrm{D}{ }^{1} \mathrm{H}$ aliphatic region of the EphA4-LBD (at $20 \mu \mathrm{M}$ ) as illustrated in Figure 1. $\Delta \delta$ : $0<-<0.1 \mathrm{ppm} ; 0.1 \mathrm{ppm}<+<0.2 \mathrm{ppm} ; 0.2<++<0.3 ;+++>0.3 \mathrm{ppm}$.

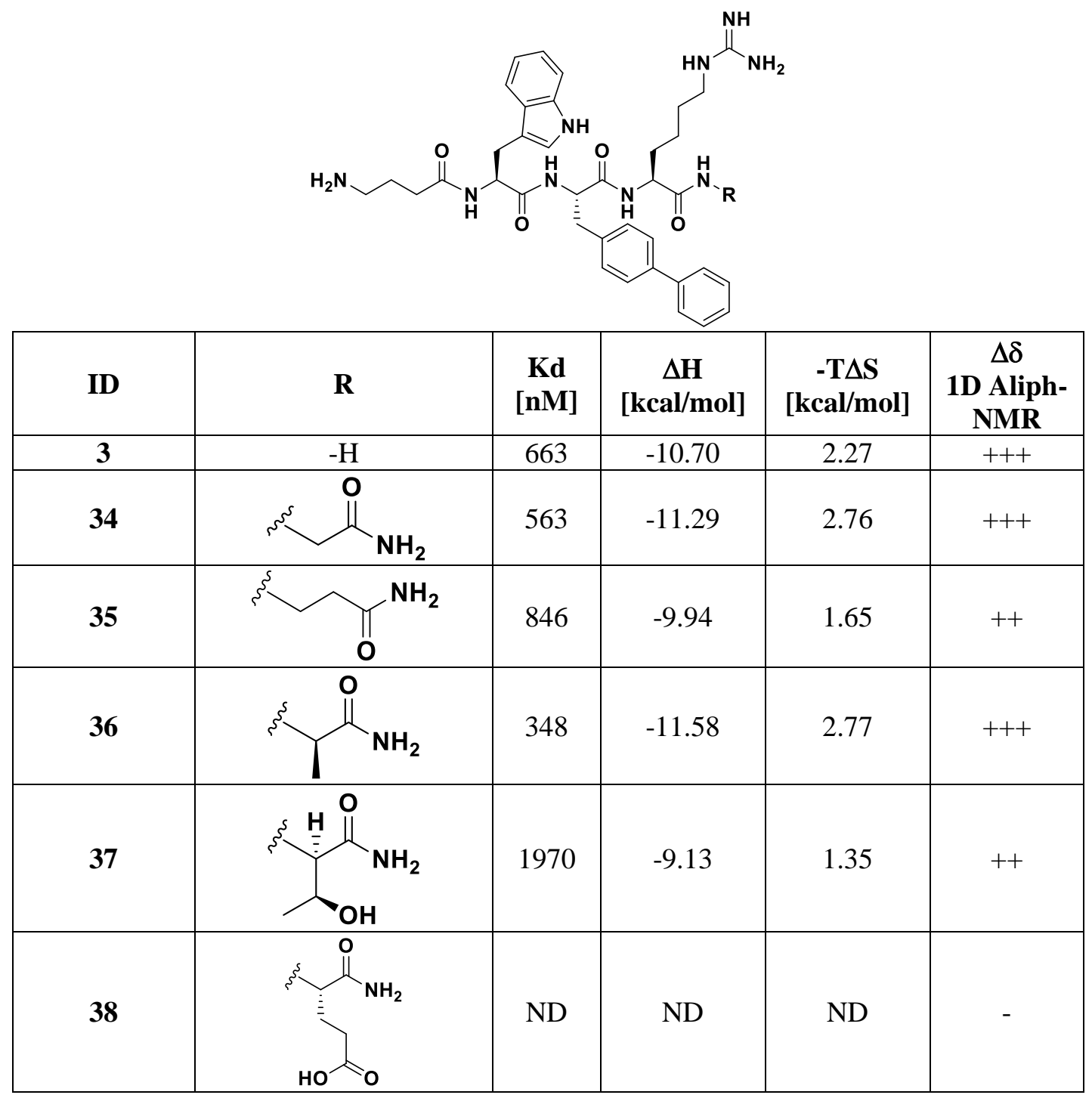




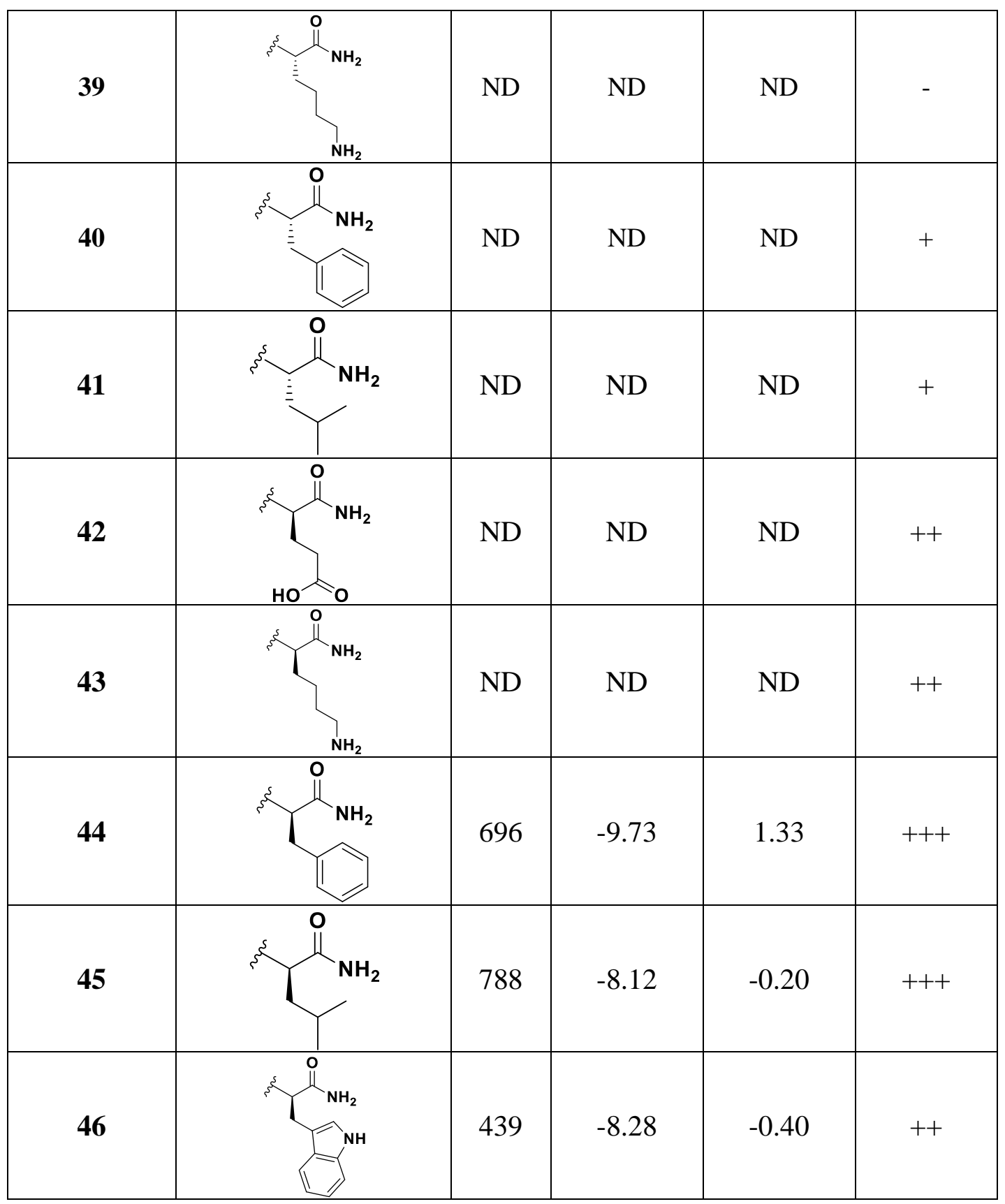


Table S2. Data collection and Refinement statistics for 150D4

\begin{tabular}{|c|c|}
\hline Data collection & \\
\hline Ligand & 150D4 \\
\hline X-ray source & P13 (DESY) \\
\hline Wavelength $[\AA]$ & 1.0332 \\
\hline Detector & PILATUS 6M \\
\hline Temperature $[\mathrm{K}]$ & 100 \\
\hline Space group & $\mathrm{P} 4_{3} 2_{1} 2$ \\
\hline \multicolumn{2}{|l|}{ Cell: } \\
\hline $\mathrm{a} ; \mathrm{b} ; \mathrm{c} ;[\AA]$ & $55.03 ; 55.03 ; 147.30$ \\
\hline$\alpha ; \beta ; \gamma ;\left[{ }^{\circ}\right]$ & $90.0 ; 90.0 ; 90.0$ \\
\hline Resolution $[\AA]$ & $1.43(1.54-1.43)$ \\
\hline Unique reflections & $34710(1736)$ \\
\hline Multiplicity & $12.9(4.0)$ \\
\hline Spherical completeness [\%] & $81.0(20.1)$ \\
\hline Ellipsoidal completeness [\%] & $93.8(57.1)$ \\
\hline $\mathrm{R}_{\mathrm{pim}}[\%]$ & $1.5(48.0)$ \\
\hline $\mathrm{R}_{\text {sym }}[\%]$ & $5.3(88.3)$ \\
\hline $\mathrm{R}_{\text {meas }}[\%]$ & $5.5(101.2)$ \\
\hline $\mathrm{CC} 1 / 2[\%]$ & $100.00(53.40)$ \\
\hline $\operatorname{Mean}(\mathrm{I}) / \mathrm{sd}$ & $24.9(1.2)$ \\
\hline \multicolumn{2}{|l|}{ Refinement statistics } \\
\hline Ligand & 150D4 \\
\hline Resolution $[\AA]$ & $51.55-1.43$ \\
\hline Number of reflections (working/test) & $32995 / 1715$ \\
\hline $\mathrm{R}_{\text {cryst }}[\%]$ & 15.3 \\
\hline $\mathrm{R}_{\text {free }}[\%]^{2}$ & 20.8 \\
\hline \multicolumn{2}{|l|}{ Total number of atoms: } \\
\hline Protein & 1517 \\
\hline Water & 303 \\
\hline Ligand & 70 \\
\hline Acetate & 4 \\
\hline \multicolumn{2}{|l|}{ Deviation from ideal geometry: } \\
\hline Bond lengths $[\AA]$ & 0.010 \\
\hline Bond angles $\left[{ }^{\circ}\right]$ & 1.64 \\
\hline Bonded B's $\left[\AA^{2}\right]$ & 3.4 \\
\hline \multicolumn{2}{|l|}{ Ramachandran plot: } \\
\hline Most favoured regions [\%] & 90.3 \\
\hline Additional allowed regions [\%] & 9.1 \\
\hline Generously allowed regions [\%] & 0.6 \\
\hline Disallowed regions [\%] & 0.0 \\
\hline
\end{tabular}


Table S3. In vivo $\mathrm{PK} /$ brain exposure studies

\begin{tabular}{|c|c|c|c|c|}
\hline Dose/route & $\begin{array}{l}\text { Plasma drug } \\
\text { concentration } \\
\text { (time point) }\end{array}$ & $\begin{array}{c}\text { Brain } \\
\text { concentration } \\
\text { (time point) }\end{array}$ & $\begin{array}{c}\text { Total extracted } \\
\text { brain } \\
\text { compound } \\
\text { (time point) }\end{array}$ & $\begin{array}{l}\text { Amount of } \\
\text { compound per mg } \\
\text { of wet brain tissue } \\
\text { (time point) }\end{array}$ \\
\hline \multirow[t]{2}{*}{$\begin{array}{l}10 \mathrm{mg} / \mathrm{kg} \\
\text { IV }\end{array}$} & $\begin{array}{c}484 \pm 250 \mathrm{ng} / \mathrm{ml} \\
(30 \mathrm{~min})\end{array}$ & $\begin{array}{l}189 \pm 1 \mathrm{ng} / \mathrm{ml} \\
(30 \mathrm{~min})\end{array}$ & $\begin{array}{l}205.6 \pm 3 \mathrm{ng} \\
\quad(30 \mathrm{~min})\end{array}$ & $\begin{array}{c}4.73 \pm 0.1 \mathrm{ng} / \mathrm{mg} \\
(30 \mathrm{~min})\end{array}$ \\
\hline & $\begin{array}{c}14.2 \pm 5.0 \mathrm{ng} / \mathrm{ml} \\
(24 \mathrm{hr})\end{array}$ & $\begin{array}{c}54 \pm 3 \mathrm{ng} / \mathrm{ml} \\
(24 \mathrm{hr})\end{array}$ & $\begin{array}{l}60 \pm 8 \mathrm{ng} \\
(24 \mathrm{hr})\end{array}$ & $\begin{array}{c}1.36 \pm 0.1 \mathrm{ng} / \mathrm{mg} \\
(24 \mathrm{hr})\end{array}$ \\
\hline \multirow[t]{2}{*}{$\begin{array}{c}50 \mathrm{mg} / \mathrm{Kg} \\
\text { IP }\end{array}$} & $\begin{array}{c}7735 \pm 120 \mathrm{ng} / \mathrm{ml} \\
(1 \mathrm{hr})\end{array}$ & $\begin{array}{c}184 \pm 87 \mathrm{ng} / \mathrm{ml} \\
(1 \mathrm{hr})\end{array}$ & $\begin{array}{c}185 \pm 38 \mathrm{ng} \\
(30 \mathrm{~min})\end{array}$ & $\begin{array}{c}4.6 \pm 2 \mathrm{ng} / \mathrm{mg} \\
(30 \mathrm{~min})\end{array}$ \\
\hline & $\begin{array}{c}45 \pm 3 \mathrm{ng} / \mathrm{ml} \\
(24 \mathrm{hr})\end{array}$ & $\begin{array}{c}45.5 \pm 0.3 \mathrm{ng} / \mathrm{ml} \\
(24 \mathrm{hr})\end{array}$ & $\begin{array}{l}34.7 \pm 6 \mathrm{ng} \\
(24 \mathrm{hr})\end{array}$ & $\begin{array}{c}1.1 \pm 0.01 \mathrm{ng} / \mathrm{mg} \\
(24 \mathrm{hr})\end{array}$ \\
\hline
\end{tabular}

Balb/C mice ( $n=4$ per route) were injected with 150D4 at the indicated doses. The formulations were generated dissolving $8.7 \mathrm{mg}$ of 150D4 in $1392 \mu \mathrm{L}$ of solvent comprised of $80 \%$ PBS, $10 \%$ Ethanol, and $10 \%$ Tween 80 to a final concentration a $6.25 \mathrm{mg} / \mathrm{mL}$ (used for the IP study) or dissolving $2.5 \mathrm{mg}$ 150D4 with $1000 \mu \mathrm{L}$ of the $80 \%$ PBS, $10 \%$ Ethanol, and $10 \%$ Tween 80 to a final concentration of $2.5 \mathrm{mg} / \mathrm{mL}$ (for the IV study). Plasma drug concentration was determined by LCMS at the indicated time points (n $=2$ mice per route). Brains were collected at the indicated time points, homogenized and extracted compound analyzed by LCMS ( $\mathrm{n}=2$ mice per route). All procedures were conducted according to IACUC approved protocols at the UCSD Pharmacology Core facility. 
Figure S1. HPLC traces, High Resolution mass, and 1D ${ }^{1} \mathrm{H}-\mathrm{NMR}$ spectra for tested compounds in Table 6. Analytical run was accomplished using Atlantis $\mathrm{T} 33 \mu \mathrm{m} 4.6 \times 150 \mathrm{~mm}\left(\mathrm{H}_{2} \mathrm{O} / \mathrm{ACN}\right.$ gradient from $5 \%$ to $100 \%$ in $45 \mathrm{~min}$ ). All compounds displayed purity $>95 \%$. High-resolution mass spectral data were acquired on an Agilent 6545 Q-TOF LC/MS instrument. NMR spectra were recorded on Bruker Avance III $700 \mathrm{MHz}$ equipped with a TCI cryo-probe.

\section{Compound 150D4}

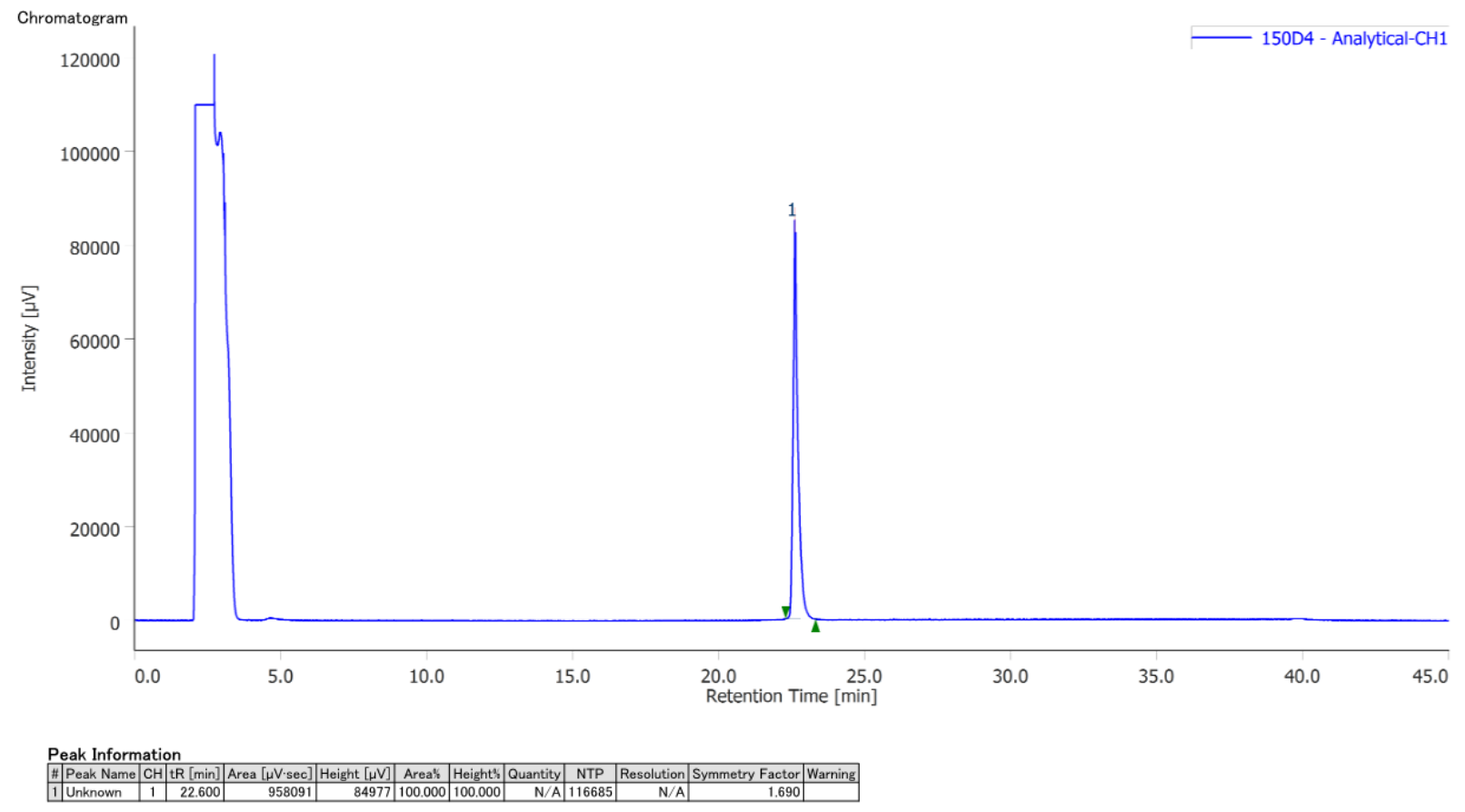




\section{Compound 31}

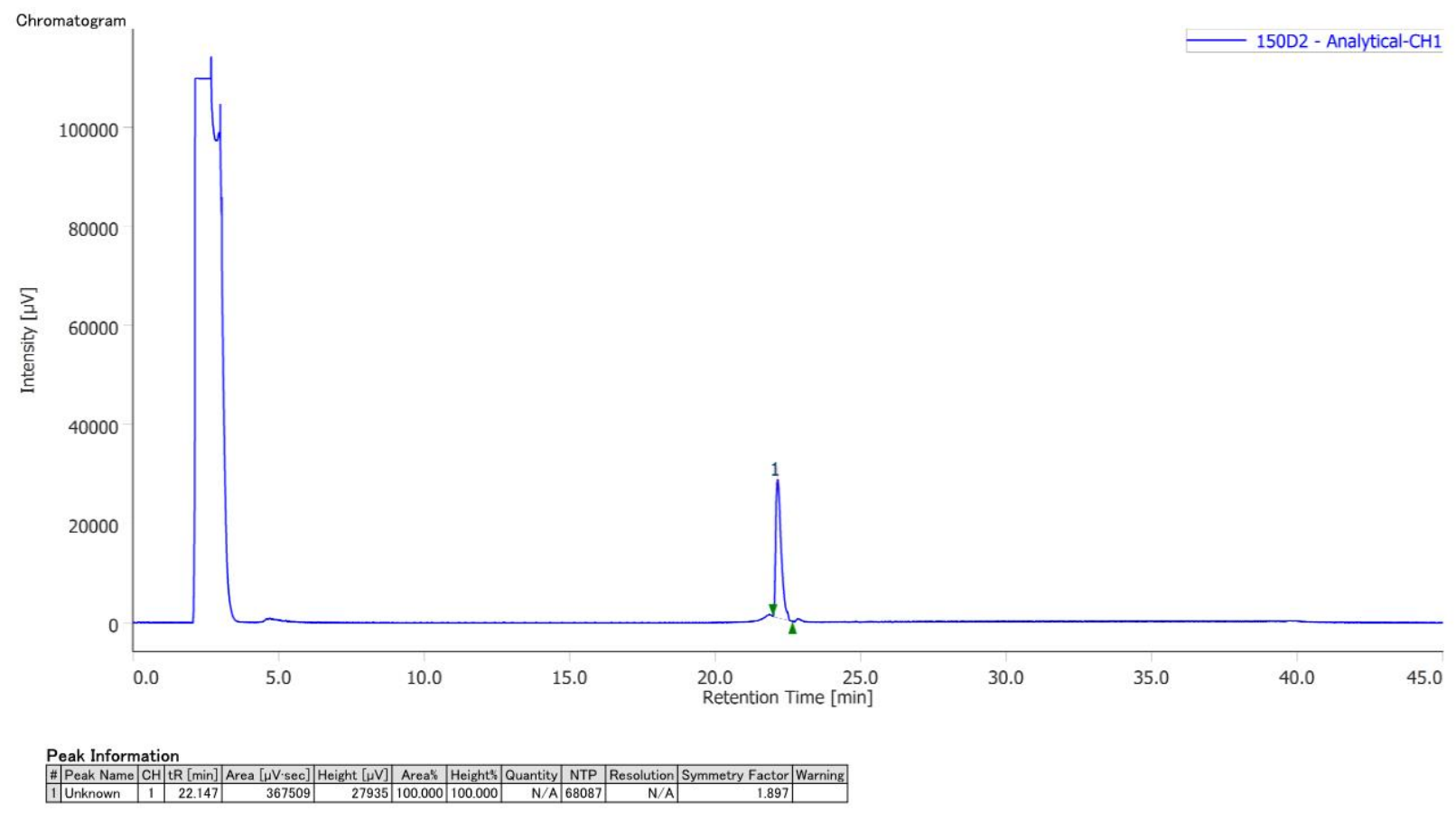

\section{Compound 30}

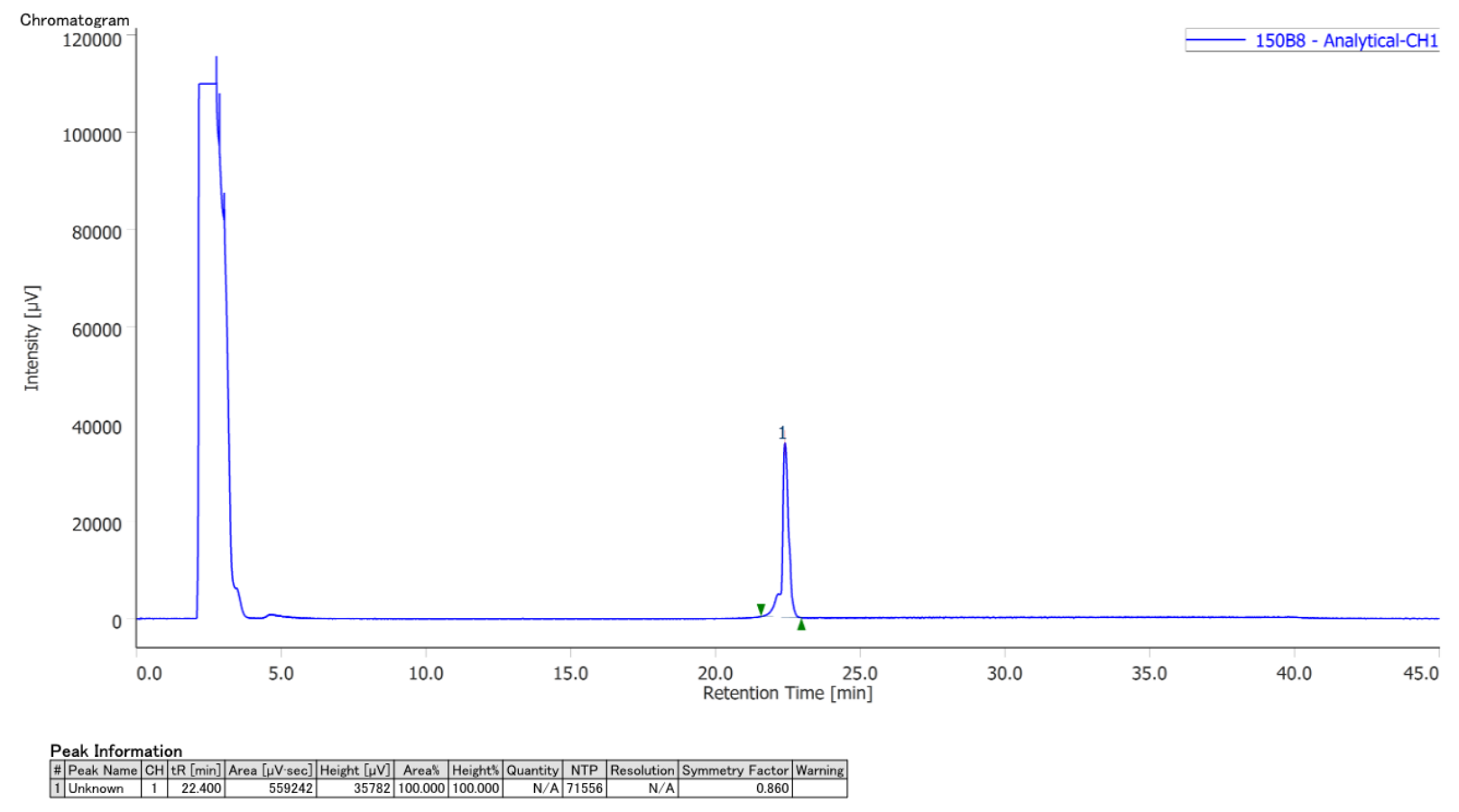




\section{Compound 32}

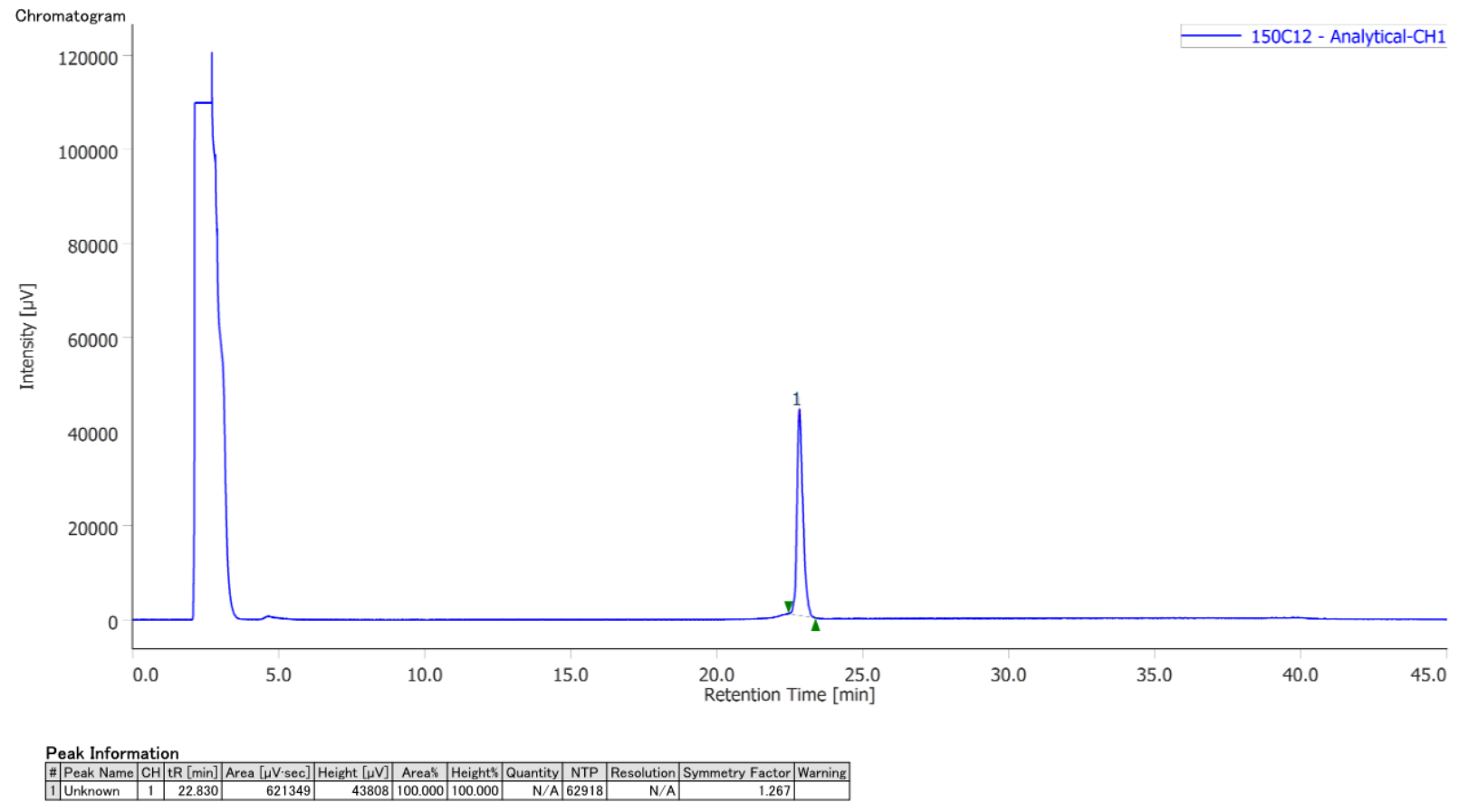

\begin{tabular}{|c|c|c|c|}
\hline ID & IUPAC name & $\begin{array}{c}\text { Calcd } \\
{[\mathrm{M}+\mathrm{H}]^{+}}\end{array}$ & $\begin{array}{c}\text { Obs. } \\
{[\mathbf{M}+\mathbf{H}]^{+}}\end{array}$ \\
\hline 30 & $\begin{array}{l}\text { (1S,3S)-3-amino-N-((S)-1-(((S)-1-(((S)-6-guanidino-1-((2-((4- } \\
\text { (morpholinomethyl)phenyl)amino)-2-oxoethyl)amino)-1- } \\
\text { oxohexan-2-yl)amino)-3-(2'-methoxy-[1,1'-biphenyl]-4-yl)-1- } \\
\text { oxopropan-2-yl)amino)-3-(5-hydroxy-1H-indol-3-yl)-1- } \\
\text { oxopropan-2-yl)cyclohexane-1-carboxamide }\end{array}$ & 1000.5404 & 1000.5412 \\
\hline 31 & $\begin{array}{l}\text { (1S,3S)-N-((S)-1-(((S)-3-([1,1'-biphenyl]-4-yl)-1-(((S)-6- } \\
\text { guanidino-1-((2-((4-(morpholinomethyl)phenyl)amino)-2- } \\
\text { oxoethyl)amino)-1-oxohexan-2-yl)amino)-1-oxopropan-2- } \\
\text { yl)amino)-3-(5-hydroxy-1H-indol-3-yl)-1-oxopropan-2-yl)-3- } \\
\text { aminocyclohexane-1-carboxamide }\end{array}$ & 970.5297 & 970.5301 \\
\hline 32 & $\begin{array}{l}\text { (1S,3S)-3-amino-N-((S)-1-(((S)-1-(((S)-6-guanidino-1-((2-((4- } \\
\text { morpholinophenyl)amino)-2-oxoethyl)amino)-1-oxohexan-2- } \\
\text { yl)amino)-3-(2'-methoxy-[1,1'-biphenyl]-4-yl)-1-oxopropan-2- } \\
\text { yl)amino)-3-(5-hydroxy-1H-indol-3-yl)-1-oxopropan-2- } \\
\text { yl)cyclohexane-1-carboxamide }\end{array}$ & 986.5246 & 986.5264 \\
\hline $\begin{array}{c}33 \\
150 D 4\end{array}$ & $\begin{array}{l}\text { (1S,3S)-N-((S)-1-(((S)-3-([1,1'-biphenyl]-4-yl)-1-(((S)-6- } \\
\text { guanidino-1-((2-((4-morpholinophenyl)amino)-2- } \\
\text { oxoethyl)amino)-1-oxohexan-2-yl)amino)-1-oxopropan-2- } \\
\text { yl)amino)-3-(5-hydroxy-1H-indol-3-yl)-1-oxopropan-2-yl)-3- } \\
\text { aminocyclohexane-1-carboxamide }\end{array}$ & 956.5140 & 956.5151 \\
\hline
\end{tabular}




\section{Compound 30}

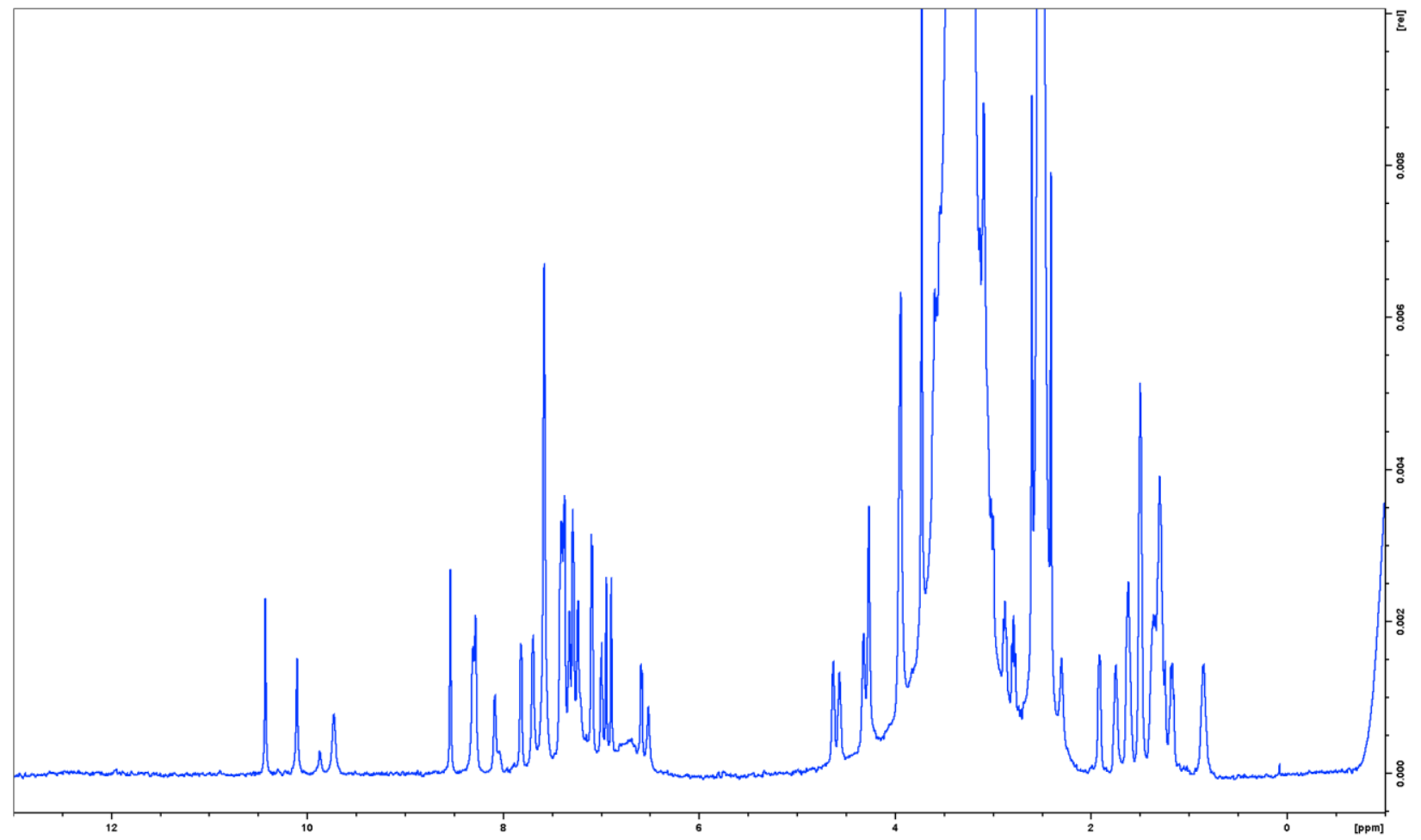

\section{Compound 31}

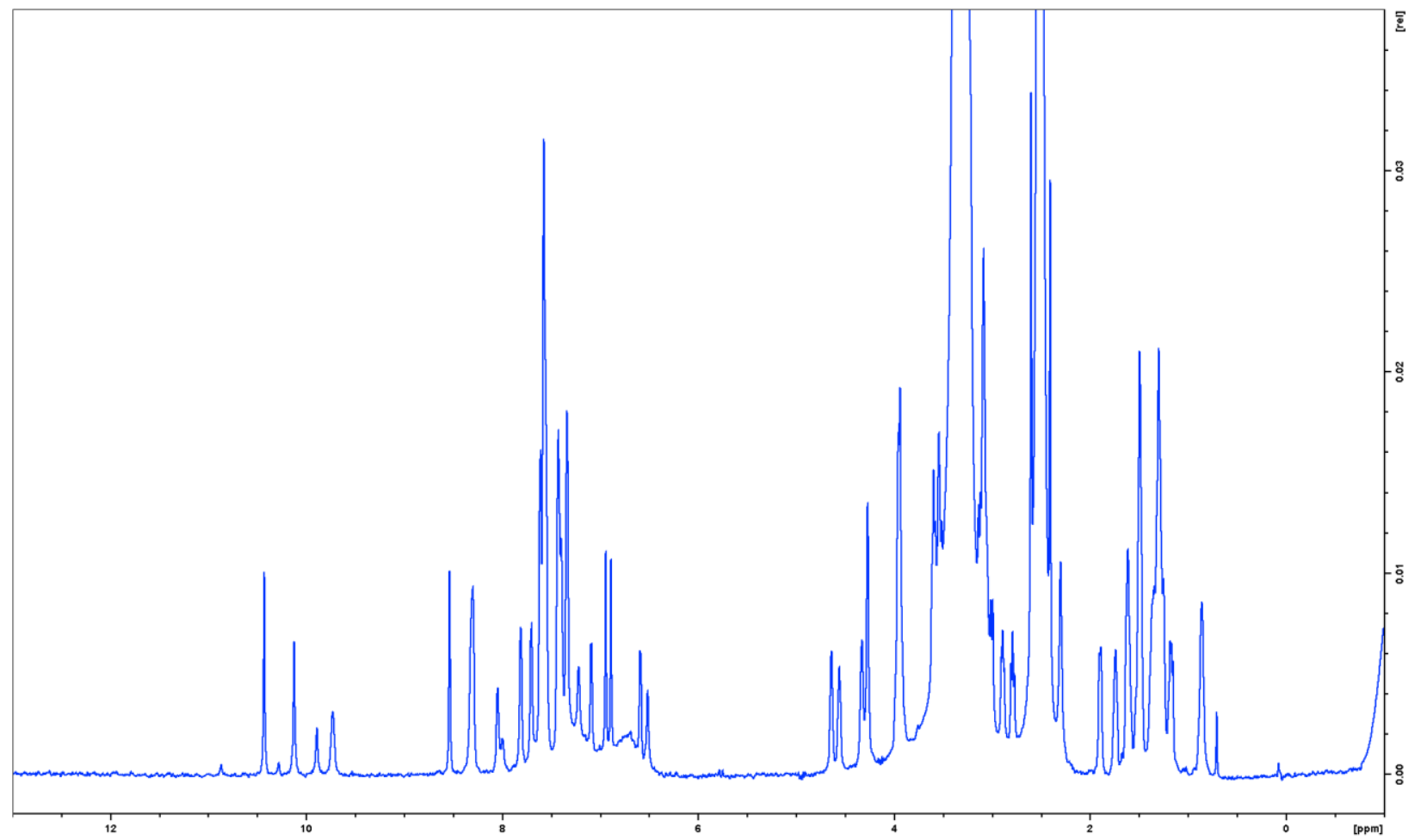




\section{Compound 32}

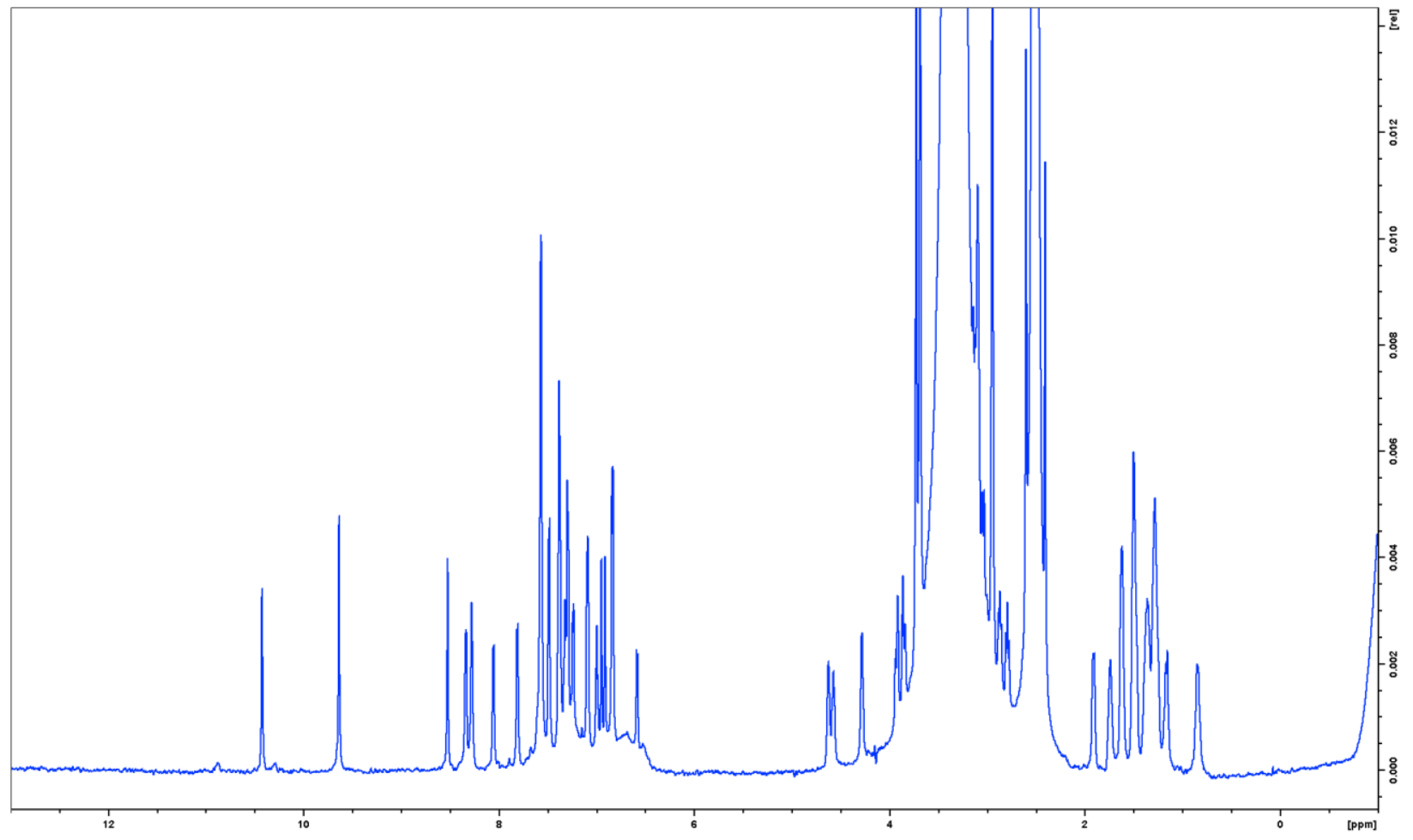

Compound 33 (150D4)

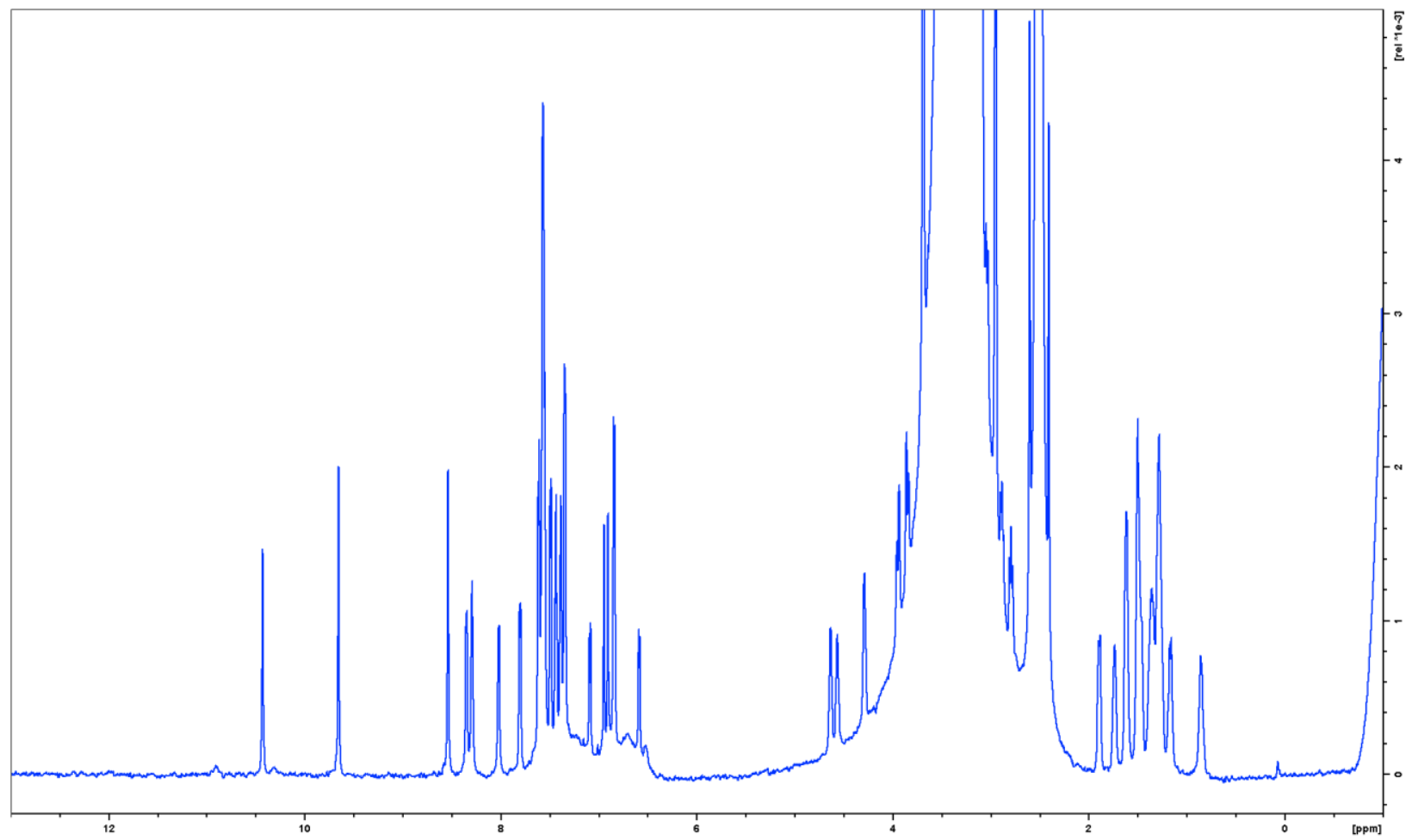


Figure S2. General scheme for the synthesis of compounds 150D4 and 32. Conditions: (a) DMF, 30 min, rt; (b) sodium triacetoxyborohydride (3 eq.), o/n, rt; (c) Fmoc-Gly-COOH (3 equiv), HATU (3 eq.), Oxyma Pure (3 eq.), DIPEA (5 eq.), 2 h, rt; (d) 20\% 4-methylpiperidine in DMF, rt; (e) Fmoc-L-

HomoArg(Pbf)-OH (3 eq.), HATU (3 eq.), Oxyma Pure (3 eq.), DIPEA (5 eq.), 1 h, rt; (f) Fmoc-pphenyl-L-phenylalanine or Fmoc-4-(2-methoxyphenyl)-L-phenylalanine (3 eq.), HATU (3 eq.), Oxyma Pure (3 eq.), DIPEA (5 eq.), 1 h, rt; (g) Fmoc-5-Hydroxy-L-tryptophan (3 eq.), HATU (3 eq.), Oxyma Pure (3 eq.), DIPEA (5 eq.), 1 h, rt; (h) (1S,3S)-3-(Boc-amino)cyclohexanecarboxylic acid (3 eq.), HATU (3 eq.), Oxyma Pure (3 eq.), DIPEA (5 eq.), 1 h, rt; (g) TFA/TIS/water/phenol (94:2:2:2), 5 h, rt.

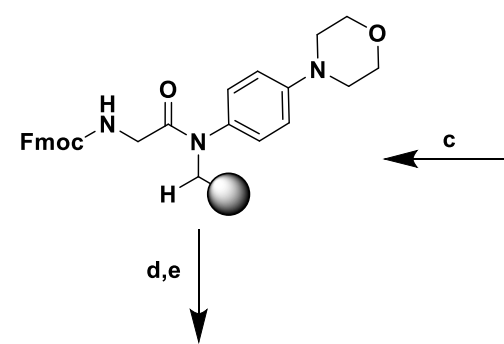

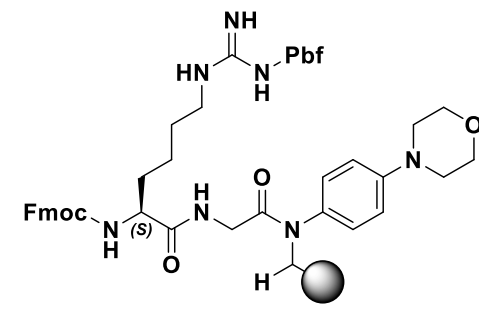

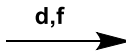

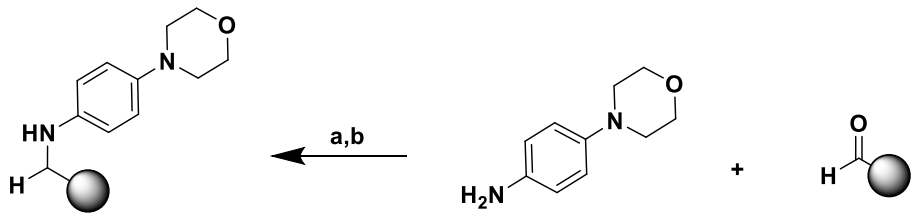<smiles>[R]c1ccccc1-c1ccc(C[C@H](NC([CH])=O)C(=O)N[C@@H](CCCCNC(=N)Nc2ccccc2)C(=O)NCC(=O)N(c2ccc(N3CCOCC3)cc2)C(C)O)cc1</smiles><smiles>[R]c1ccccc1-c1ccc(C[C@@H](NC(=O)[C@H](CCCCNC(=N)NCc2ccccc2)NC(=O)CNC(=O)N(C(=O)[C@H](C)O)c2ccc(N3CCOCC3)cc2)NC(=O)[C@H](Cc2c[nH]c3ccc(O)cc23)NC(=O)[C@H](C)O)cc1</smiles><smiles>[R]c1ccccc1-c1ccc(C[C@H](NC(=O)[C@H](Cc2c[nH]c3ccc(O)cc23)NC(=O)[C@@H]2CCC[C@@H](N)C2)C(=O)N[C@@H](CCCCNC(=N)N)C(=O)NCC(=O)Nc2ccc(N3CCOCC3)cc2)cc1</smiles>

$R=H$ for $150 D 4$

$R=$ OMe for 32 
Figure S3. General scheme for the synthesis of compounds 31 and 30. Conditions: (a) DMF, 30 min, rt; (b) sodium triacetoxyborohydride ( 3 eq.), o/n, rt; (c) Fmoc-Gly-COOH (3 equiv), HATU (3 eq.), Oxyma Pure (3 eq.), DIPEA (5 eq.), 2 h, rt; (d) 20\% 4-methylpiperidine in DMF, rt; (e) Fmoc-L-HomoArg(Pbf)OH (3 eq.), HATU (3 eq.), Oxyma Pure (3 eq.), DIPEA (5 eq.), 1 h, rt; (f) Fmoc-p-phenyl-L-phenylalanine or Fmoc-4-(2-methoxyphenyl)-L-phenylalanine (3 eq.), HATU (3 eq.), Oxyma Pure (3 eq.), DIPEA (5 eq.), 1 h, rt; (g) Fmoc-5-Hydroxy-L-tryptophan (3 eq.), HATU (3 eq.), Oxyma Pure (3 eq.), DIPEA (5 eq.), 1 h, rt; (h) (1S,3S)-3-(Boc-amino)cyclohexanecarboxylic acid (3 eq.), HATU (3 eq.), Oxyma Pure (3 eq.), DIPEA (5 eq.), 1 h, rt; (g) TFA/TIS/water/phenol (94:2:2:2), 5 h, rt.

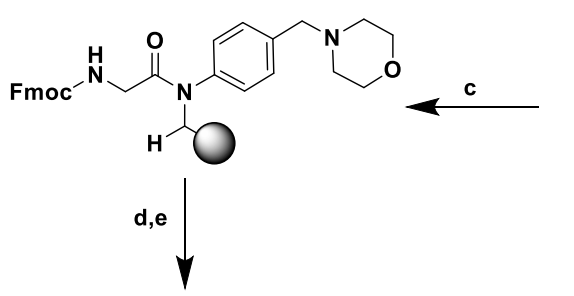<smiles>N=C(NCCCC[C@H](NC(=O)NCC(F)(F)F)C(=O)NC(=O)CO)NCc1ccccc1</smiles><smiles>[R]c1ccccc1-c1ccc(C[C@H](NC(=O)[C@H](Cc2c[nH]c3ccc(O)cc23)NC(=O)[C@@H]2CCC[C@@H](NC(=O)OC(C)(C)C)C2)C(=O)N[C@@H](CCCCNC(=N)Nc2ccccc2)C(=O)NCC(=O)N(c2ccc(CN3CCOCC3)cc2)[C@@H](C)O)cc1</smiles><smiles>[R]c1ccccc1-c1ccc(C[C@H](NC(=O)[C@H](Cc2c[nH]c3ccc(O)cc23)NC(=O)[C@@H]2CCC[C@@H](N)C2)NC(=O)[C@H](CCCCNC(=N)N)C(=O)NCC(=O)Nc2ccc(CN3CCOCC3)cc2)cc1</smiles>

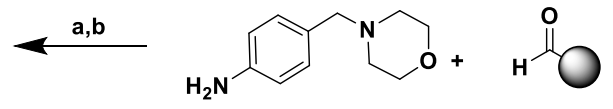<smiles>[R]c1ccccc1-c1ccc(C[C@H](NC(=O)O)C(=O)N[C@@H](CCCCNC(=N)NCc2ccccc2)C(=O)NCC(=O)N(C(=O)[C@H](C)O)c2ccc(CN3CCOCC3)cc2)cc1</smiles>

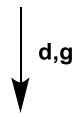

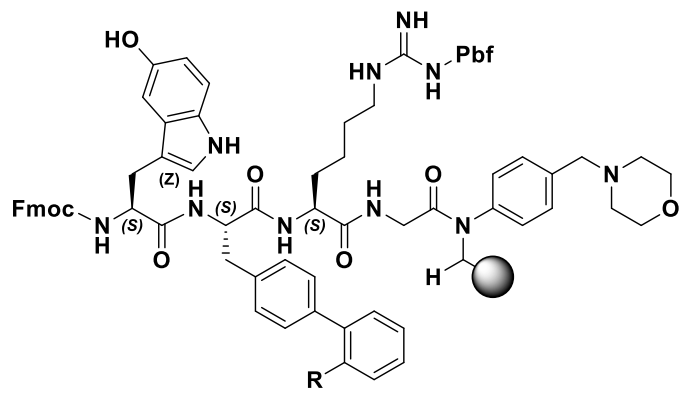

$R=\mathbf{H}$ for 31

$R=$ OMe for 30 
Figure S4. General synthetic scheme, high-resolution mass, and $1 \mathrm{D}^{\mathbf{1}} \mathrm{H}$ NMR spectra for compounds in Table 1. Conditions: (a) 20\% 4-methylpiperidine in DMF, rt. (b) Fmoc-L-HomoArg(Pbf)-OH (3 eq.), HATU (3 eq.), Oxyma Pure (3 eq.), DIPEA (5 eq.), 1 h, rt. (c) Fmoc-p-phenyl-L-phenylalanine (3 eq.), HATU (3 eq.), Oxyma Pure (3 eq.), DIPEA (5 eq.), 1 h, rt. (d) Fmoc-L-Trp(Boc) (3 eq.) for compounds 1 , 2, 3 and Fmoc-5-Hydroxy-L-tryptophan (3 eq.) for compounds 4, 5, 6, 7, 8, HATU (3 eq.), Oxyma Pure (3 eq.), DIPEA (5 eq.), 1 h, rt. (e) $\mathrm{R}_{2}$ : Boc-L-Ala (3 eq.) for compound $\mathbf{1}$; Boc- $\beta$-Ala (3 eq.) for compound 2; $\gamma$-(Boc-amino)butyric acid (3 eq.) for compound 3 and 4; (1R,3R)-3-(Boc-amino)cyclohexanecarboxylic acid (3 eq.) for compound 5; (1S,3S)-3-(Boc-amino)cyclohexanecarboxylic acid (3 eq.) for compound $\mathbf{6}$; (1R,3R)-N-Boc-3-aminocyclopentanecarboxylic acid (3 eq.) for compound 7; (1S,3S)-N-Boc-3aminocyclopentanecarboxylic acid (3 eq.) for compound 8, HATU (3 eq.), Oxyma Pure (3 eq.), DIPEA (5 eq.), $1 \mathrm{~h}$, rt. (f) TFA/TIS/water/phenol (94:2:2:2), 5 h, rt. High-resolution mass spectral data were acquired on an Agilent 6545 Q-TOF LC/MS instrument. NMR spectra were recorded on Bruker Avance III $700 \mathrm{MHz}$ equipped with a TCI cryo-probe.
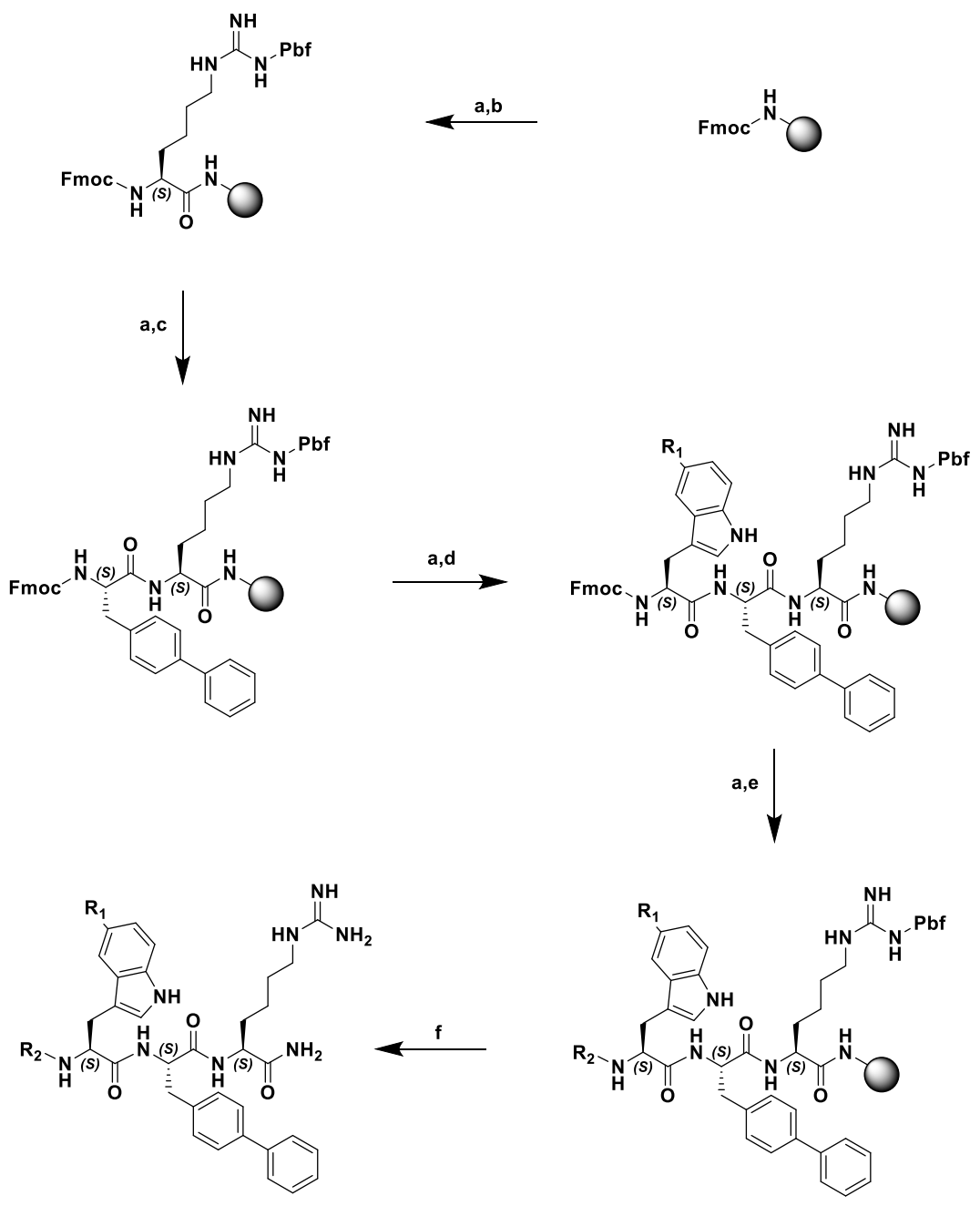

$R_{1}=H$ for $1,2,3$

$R_{1}=O H$ for $4,5,6,7,8$ 


\begin{tabular}{|c|l|c|c|}
\hline ID & \multicolumn{1}{|c|}{ IUPAC name } & \multicolumn{1}{|c|}{$\begin{array}{c}\text { Calcd } \\
{[\mathbf{M + H}]^{+}}\end{array}$} & $\begin{array}{c}\text { Obs. } \\
{[\mathbf{M + H}]^{+}}\end{array}$ \\
\hline $\mathbf{1}$ & $\begin{array}{l}\text { (S)-2-((S)-3-([1,1'-biphenyl]-4-yl)-2-((S)-2-((S)-2- } \\
\text { aminopropanamido)-3-(1H-indol-3- } \\
\text { yl)propanamido)propanamido)-6-guanidinohexanamide }\end{array}$ & 668.3668 & 668.3675 \\
\hline $\mathbf{2}$ & $\begin{array}{l}\text { (S)-2-((S)-3-([1,1'-biphenyl]-4-yl)-2-((S)-2-(3- } \\
\text { aminopropanamido)-3-(1H-indol-3- } \\
\text { yl)propanamido)propanamido)-6-guanidinohexanamide }\end{array}$ & 668.3668 & 668.3672 \\
\hline $\mathbf{3}$ & $\begin{array}{l}\text { (S)-2-((S)-3-([1,1'-biphenyl]-4-yl)-2-((S)-2-(4- } \\
\text { aminobutanamido)-3-(1H-indol-3- } \\
\text { yl)propanamido)propanamido)-6-guanidinohexanamide }\end{array}$ & 682.3824 & 682.3831 \\
\hline $\mathbf{4}$ & $\begin{array}{l}\text { (S)-2-((S)-3-([1,1'-biphenyl]-4-yl)-2-((S)-2-(4- } \\
\text { aminobutanamido)-3-(5-hydroxy-1H-indol-3- } \\
\text { yl)propanamido)propanamido)-6-guanidinohexanamide }\end{array}$ & 698.3789 \\
\hline $\mathbf{5}$ & $\begin{array}{l}\text { (1R,3R)-N-((S)-1-((S)-3-([1,1'-biphenyl]-4-yl)-1-((S)-1- } \\
\text { amino-6-guanidino-1-oxohexan-2-yl)amino)-1-oxopropan-2- } \\
\text { yl)amino)-3-(5-hydroxy-1H-indol-3-yl)-1-oxopropan-2-yl)-3- } \\
\text { aminocyclohexane-1-carboxamide }\end{array}$ & 738.4086 & 738.4095 \\
\hline $\mathbf{6}$ & $\begin{array}{l}\text { (1S,3S)-N-((S)-1-(((S)-3-([1,1'-biphenyl]-4-yl)-1-(((S)-1- } \\
\text { amino-6-guanidino-1-oxohexan-2-yl)amino)-1-oxopropan-2- } \\
\text { yl)amino)-3-(5-hydroxy-1H-indol-3-yl)-1-oxopropan-2-yl)-3- } \\
\text { aminocyclohexane-1-carboxamide }\end{array}$ & 738.4086 & 738.4103 \\
\hline $\mathbf{7}$ & $\begin{array}{l}\text { (1R,3R)-N-((S)-1-(((S)-3-([1,1'-biphenyl]-4-yl)-1-(((S)-1- } \\
\text { amino-6-guanidino-1-oxohexan-2-yl)amino)-1-oxopropan-2- } \\
\text { yl)amino)-3-(5-hydroxy-1H-indol-3-yl)-1-oxopropan-2-yl)-3- } \\
\text { aminocyclopentane-1-carboxamide }\end{array}$ & $\begin{array}{l}\text { (1S,3S)-N-((S)-1-((S)-3-([1,1'-biphenyl]-4-yl)-1-(((S)-1- } \\
\text { amino-6-guanidino-1-oxohexan-2-yl)amino)-1-oxopropan-2- } \\
\text { yl)amino)-3-(5-hydroxy-1H-indol-3-yl)-1-oxopropan-2-yl)-3- } \\
\text { aminocyclopentane-1-carboxamide }\end{array}$ & 724.3930 \\
\hline
\end{tabular}




\section{Compound 1}

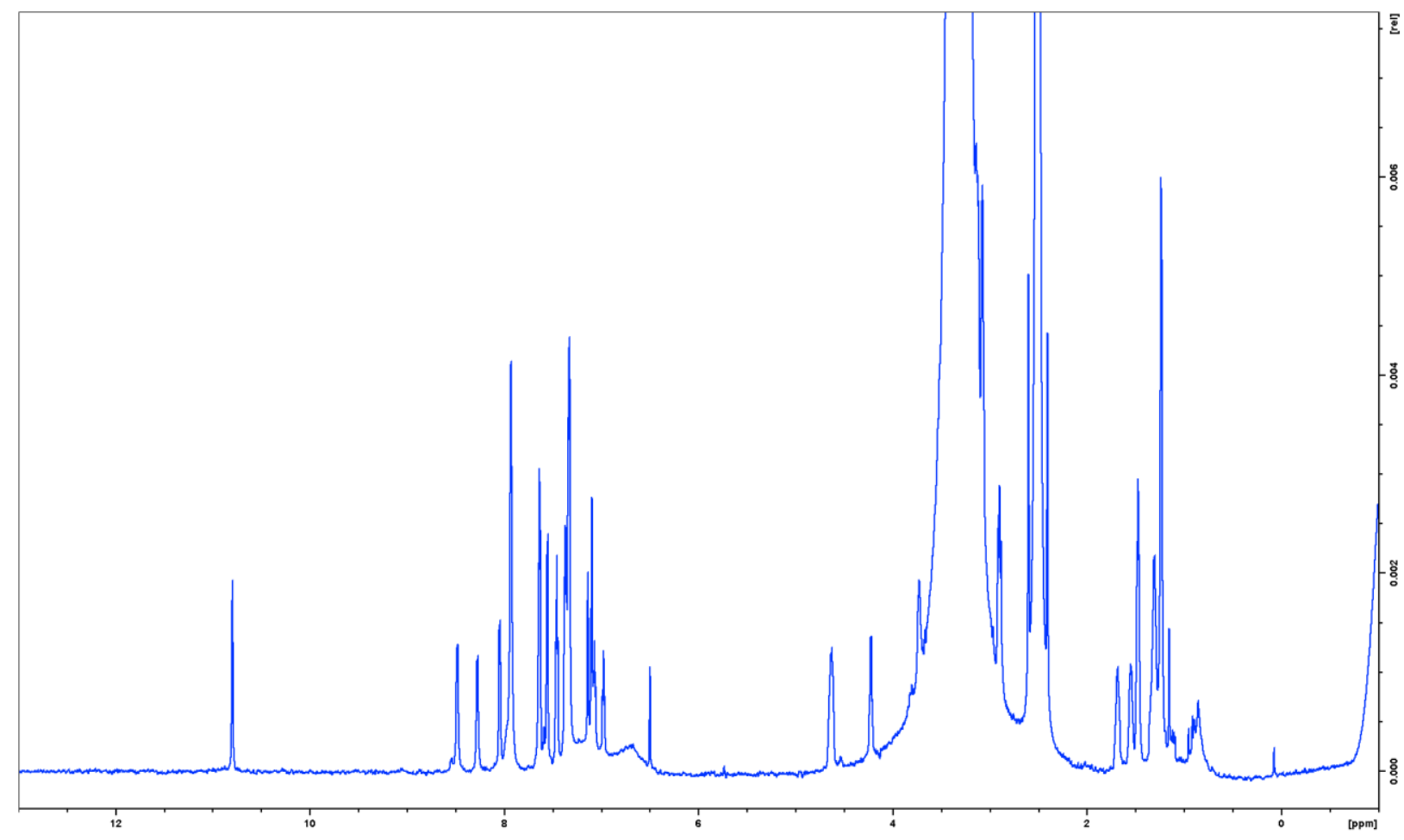

\section{Compound 2}

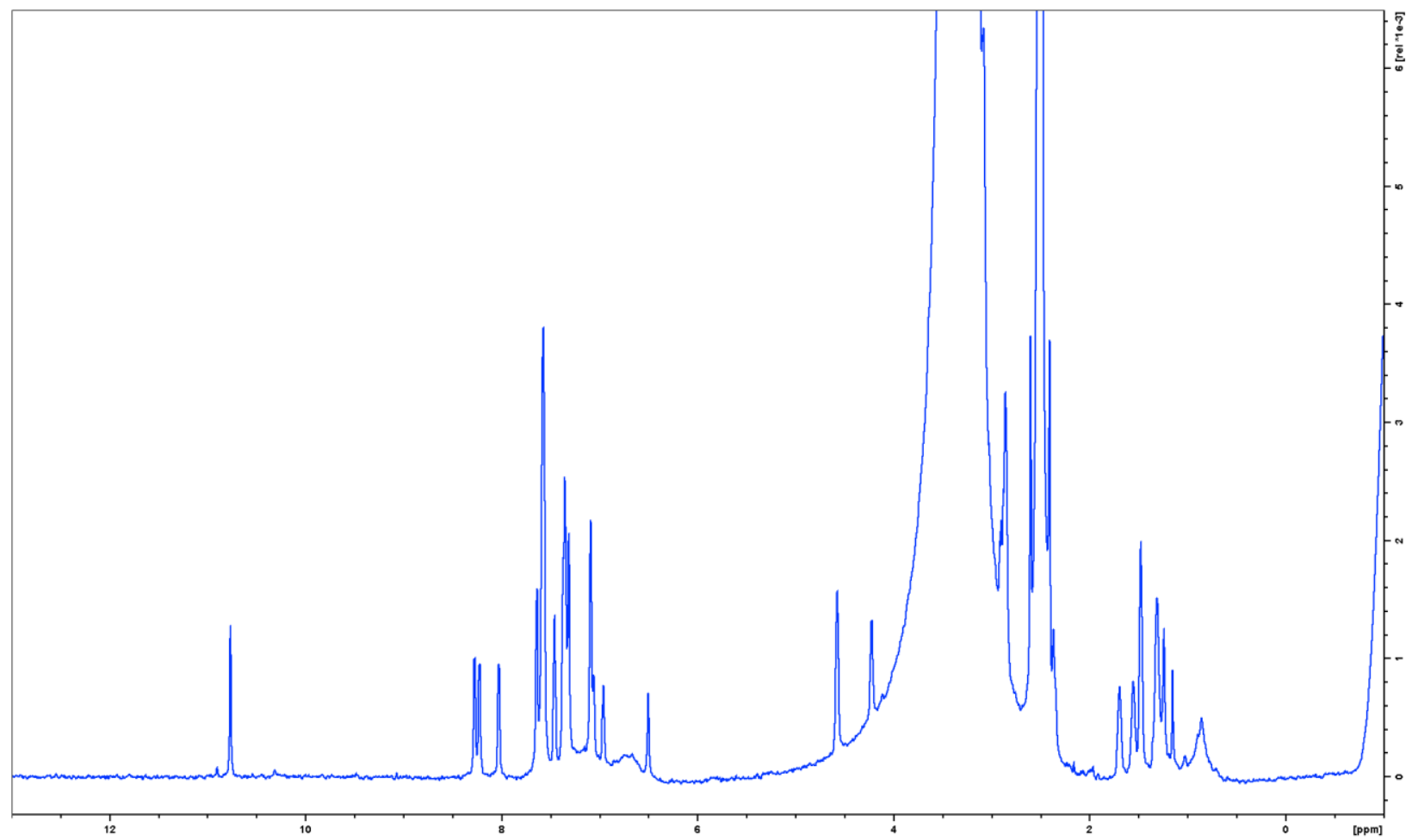


Compound 3

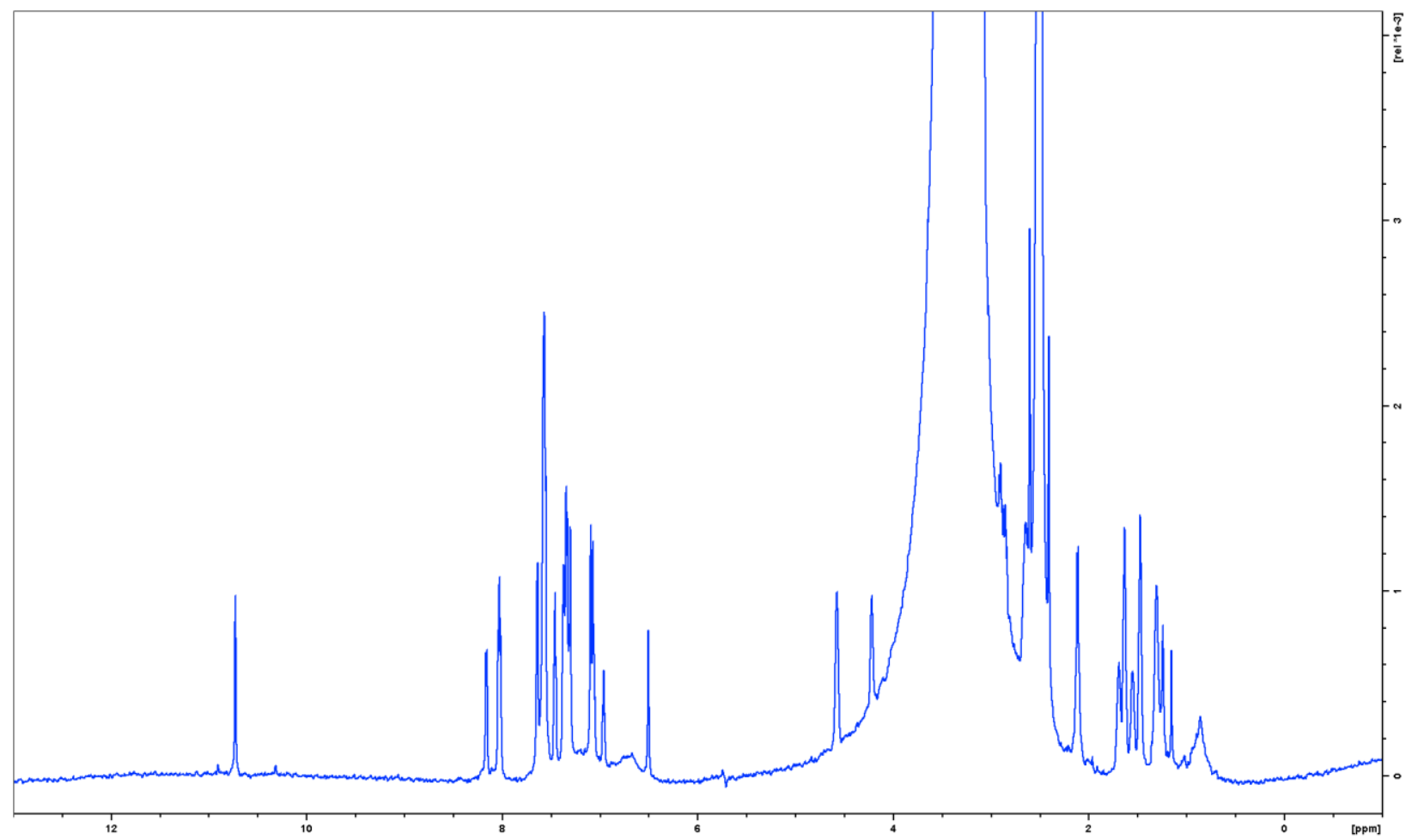

Compound 4

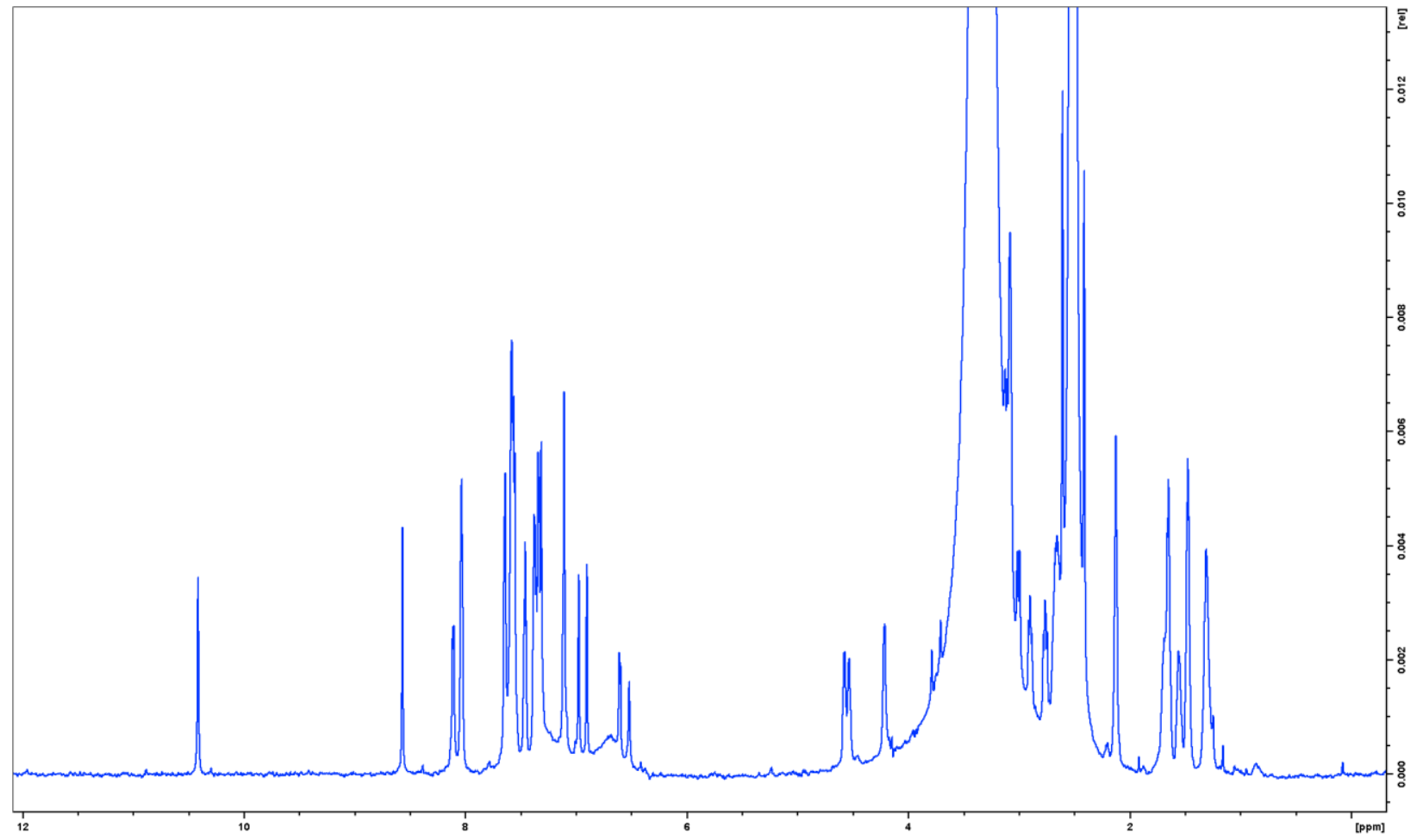




\section{Compound 5}

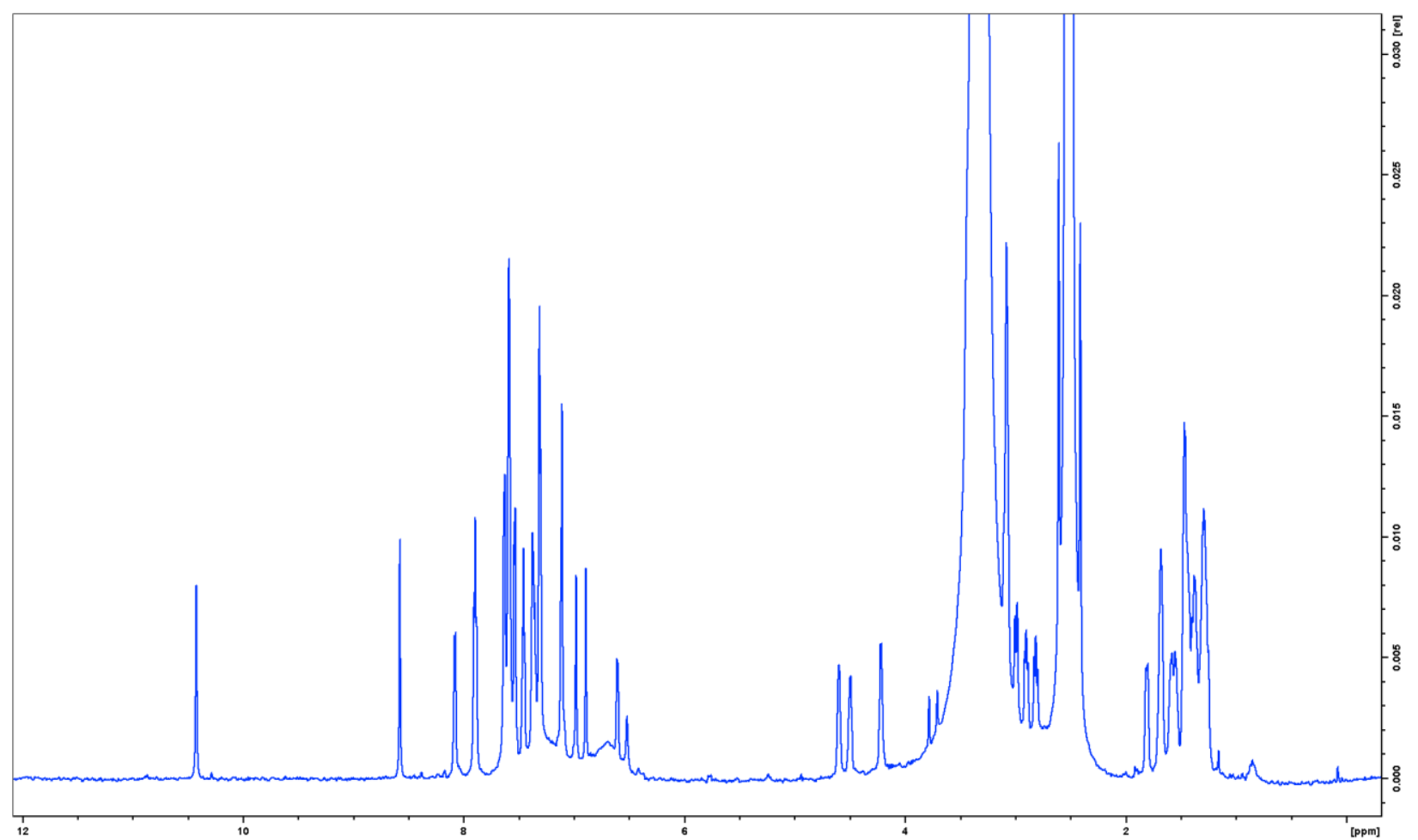

Compound 6

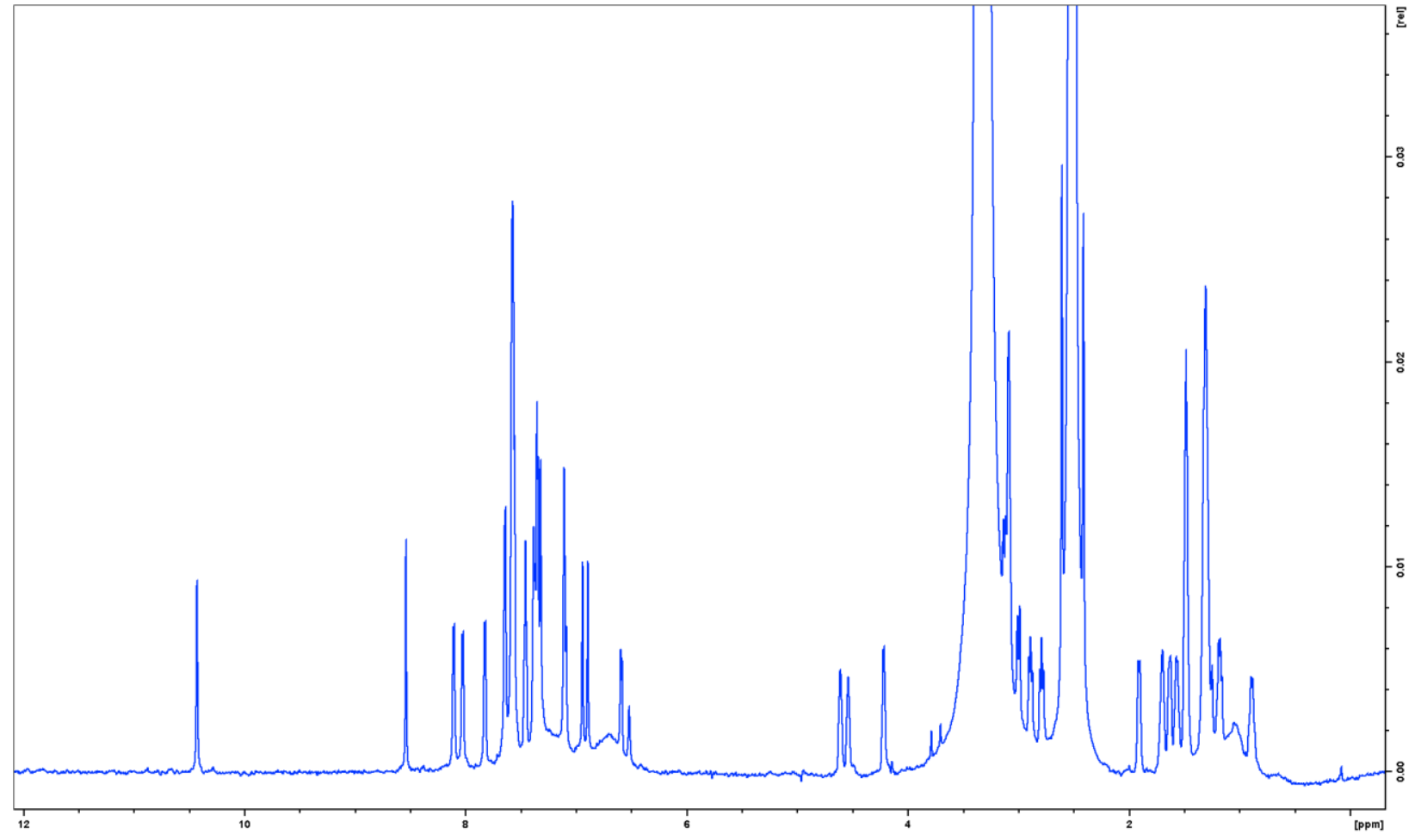




\section{Compound 7}

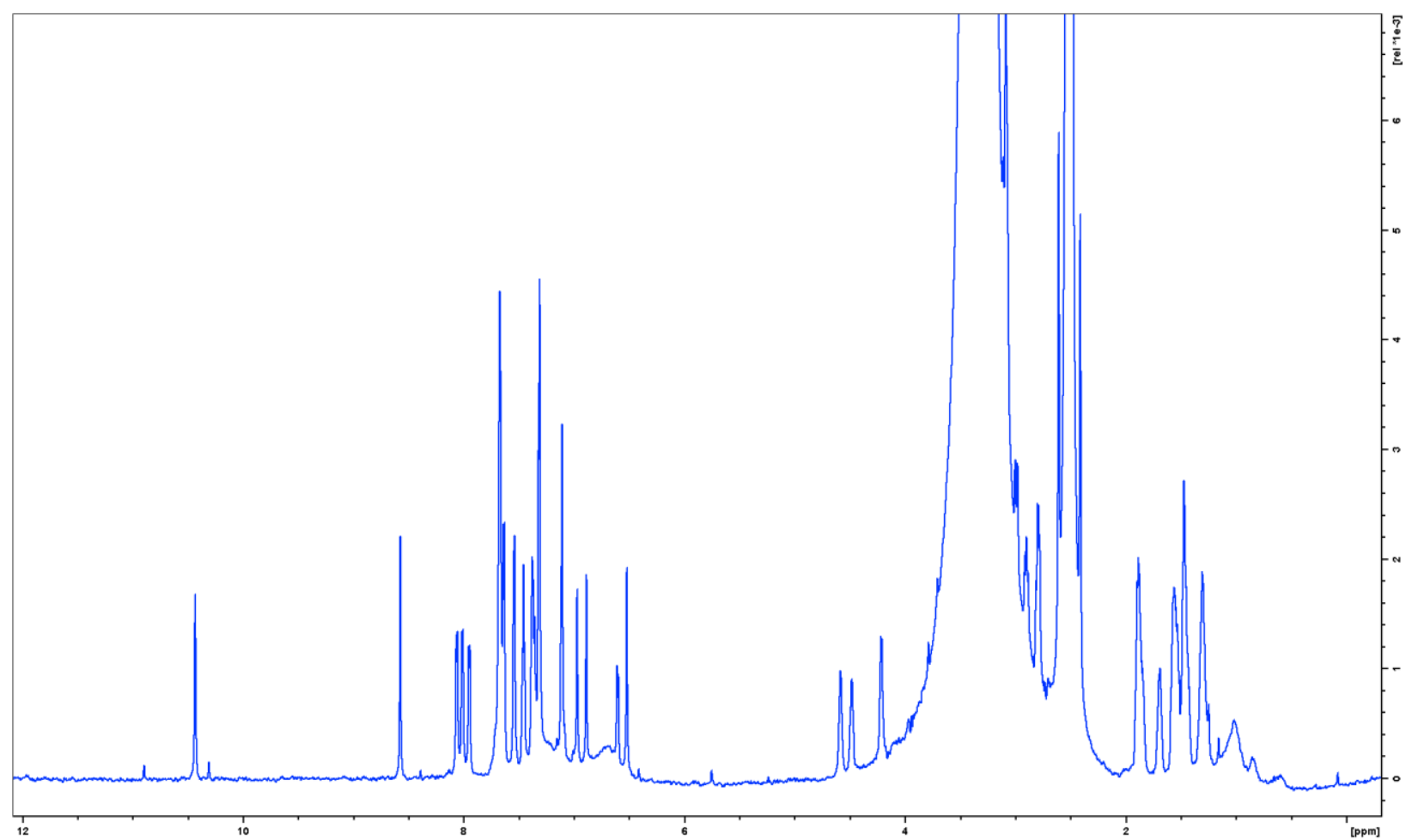

\section{Compound 8}

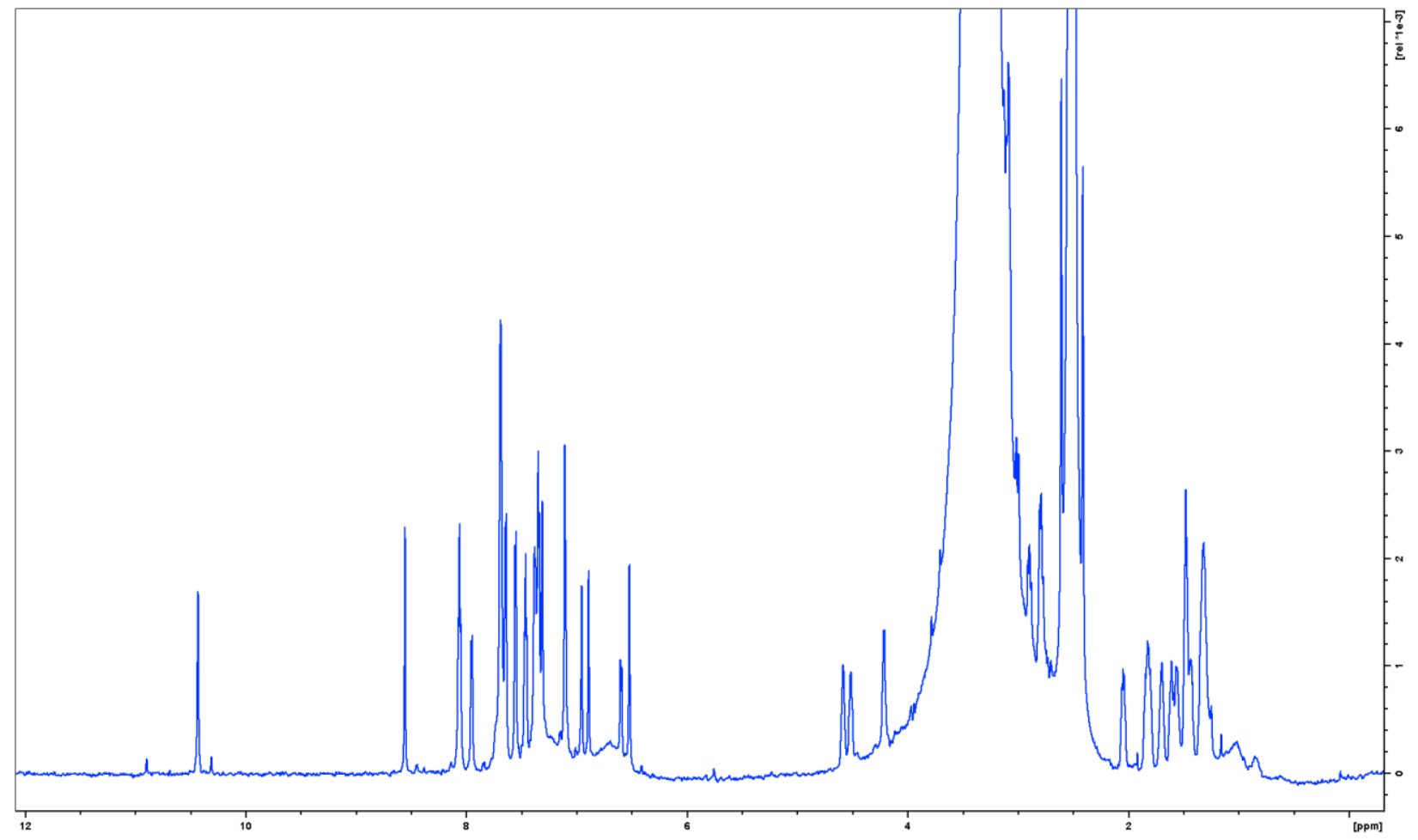


Figure S5. General synthetic scheme, high-resolution mass, and $1 \mathrm{D}^{\mathbf{1}} \mathrm{H}$ NMR spectra for compounds in Table 2. Conditions: (a) 20\% 4-methylpiperidine in DMF, rt. (b) Fmoc-L-HomoArg(Pbf)-OH (3 eq.), HATU (3 eq.), Oxyma Pure (3 eq.), DIPEA (5 eq.), 1 h, rt. (c) Fmoc-p-phenyl-L-phenylalanine (3 eq.), HATU (3 eq.), Oxyma Pure (3 eq.), DIPEA (5 eq.), 1 h, rt. (d) R: Fmoc-5-methoxy-L-Trp (3 eq.) for compound 9, Fmoc-6-methoxy-L-Trp (3 eq.) for compound 10, and Fmoc-7-aza-L-tryptophan (3 eq.) for compound 11, HATU (3 eq.), Oxyma Pure (3 eq.), DIPEA (5 eq.), 1 h, rt. (e) $\gamma$-(Boc-amino)butyric acid (3 eq.), HATU (3 eq.), Oxyma Pure (3 eq.), DIPEA (5 eq.), 1 h, rt. (f) TFA/TIS/water/phenol (94:2:2:2), 5 h, rt. High-resolution mass spectral data were acquired on an Agilent 6545 Q-TOF LC/MS instrument. NMR spectra were recorded on Bruker Avance III $700 \mathrm{MHz}$ equipped with a TCI cryoprobe.
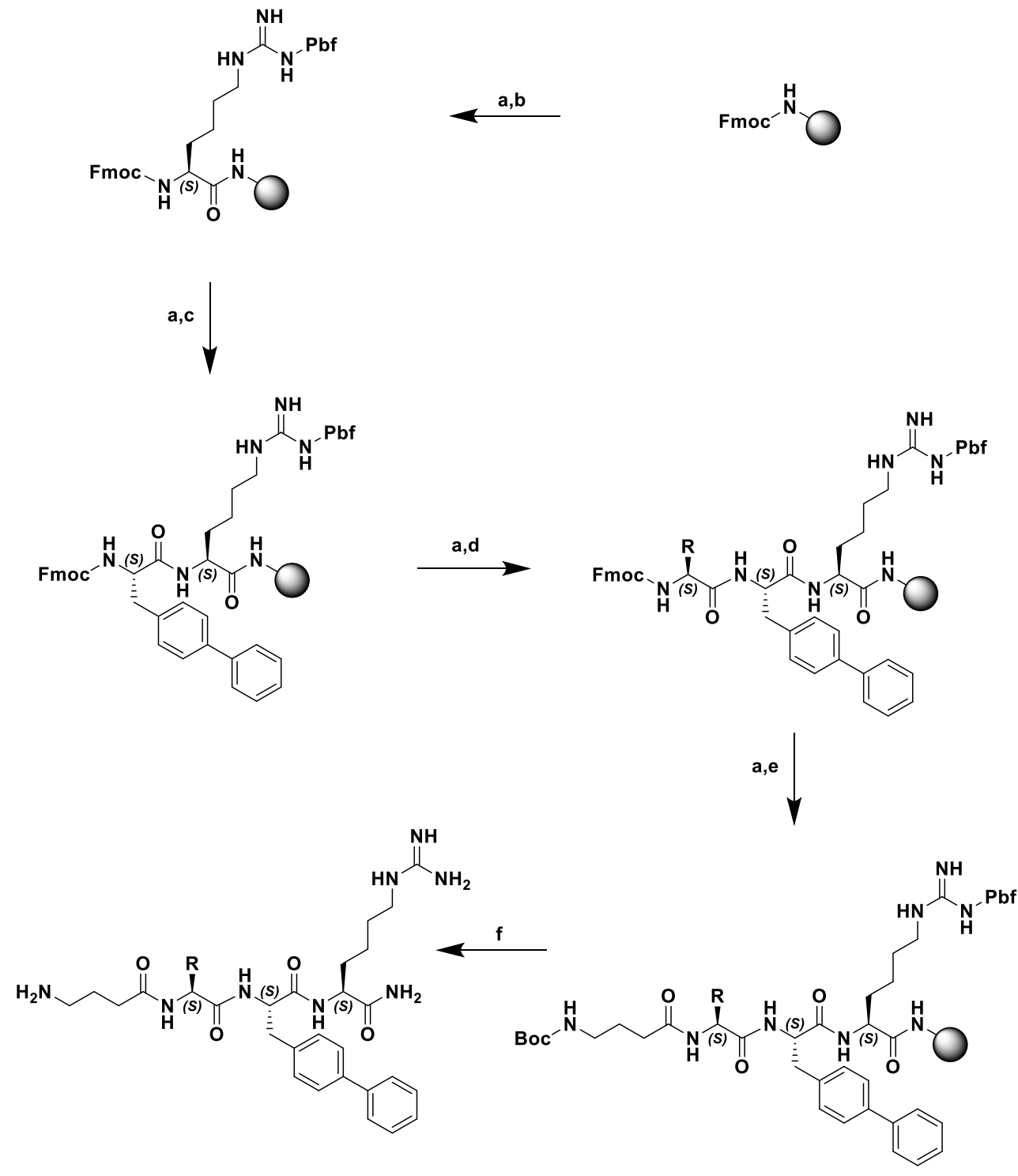


\begin{tabular}{|c|l|c|c|}
\hline $\mathbf{1 D}$ & \multicolumn{1}{|c|}{ IUPAC name } & $\begin{array}{c}\text { Calcd } \\
{[\mathbf{M}+\mathbf{H}]^{+}}\end{array}$ & $\begin{array}{c}\text { Obs. } \\
{[\mathbf{M + H}]^{+}}\end{array}$ \\
\hline $\mathbf{9}$ & $\begin{array}{l}\text { (S)-2-((S)-3-([1,1'-biphenyl]-4-yl)-2-((S)-2-(4- } \\
\text { aminobutanamido)-3-(5-methoxy-1H-indol-3- } \\
\text { yl)propanamido)propanamido)-6-guanidinohexanamide }\end{array}$ & 712.3930 & 712.3935 \\
\hline $\mathbf{1 0}$ & $\begin{array}{l}\text { (S)-2-((S)-3-([1,1'-biphenyl]-4-yl)-2-((S)-2-(4- } \\
\text { aminobutanamido)-3-(6-methoxy-1H-indol-3- } \\
\text { yl)propanamido)propanamido)-6-guanidinohexanamide }\end{array}$ & 712.3930 & 712.3946 \\
\hline $\mathbf{1 1}$ & $\begin{array}{l}\text { (S)-2-((S)-3-([1,1'-biphenyl]-4-yl)-2-((S)-2-(4- } \\
\text { aminobutanamido)-3-(1H-pyrrolo[2,3-b]pyridin-3- } \\
\text { yl)propanamido)propanamido)-6-guanidinohexanamide }\end{array}$ & 683.3777 & 683.3789 \\
\hline
\end{tabular}

\section{Compound 9}

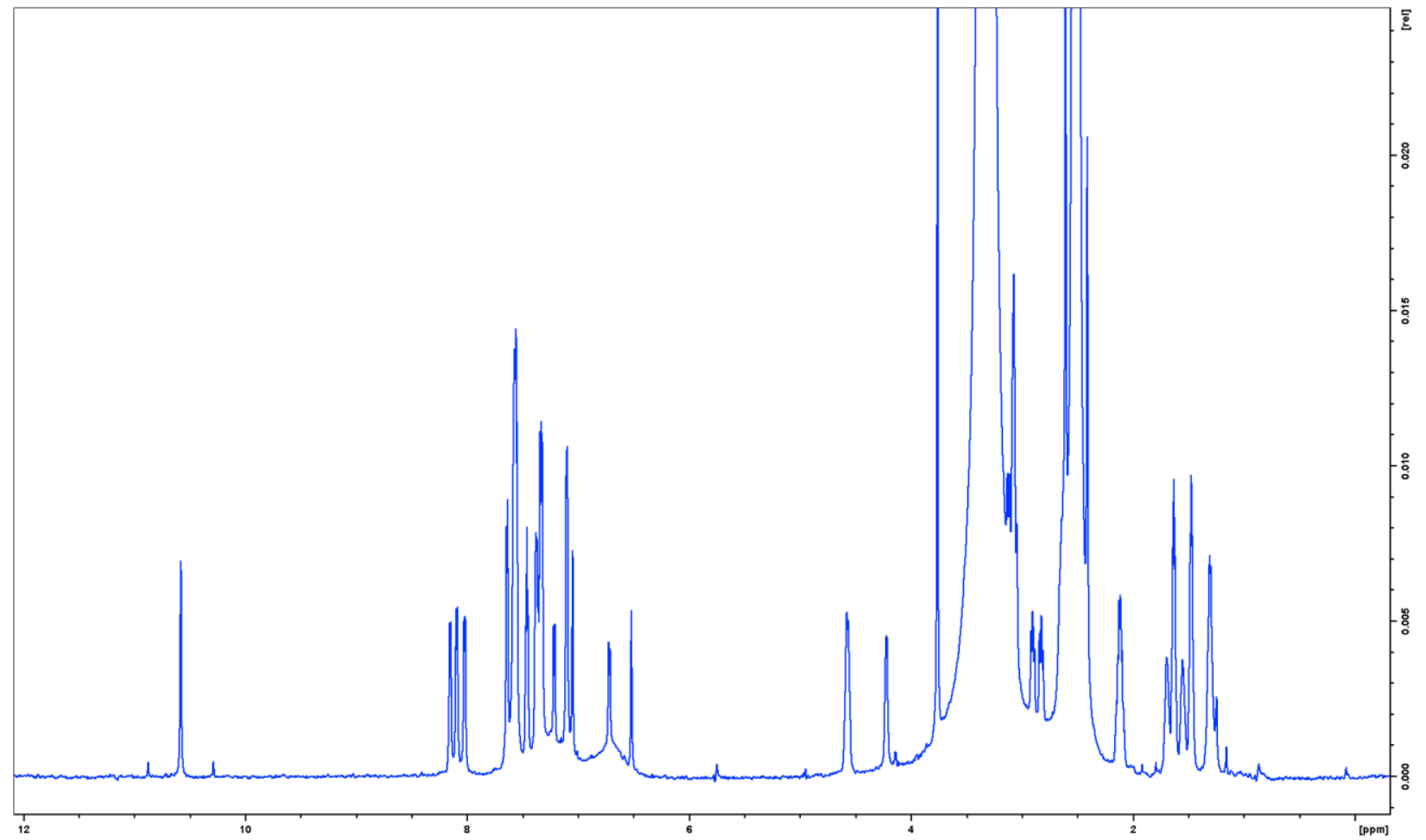




\section{Compound 10}

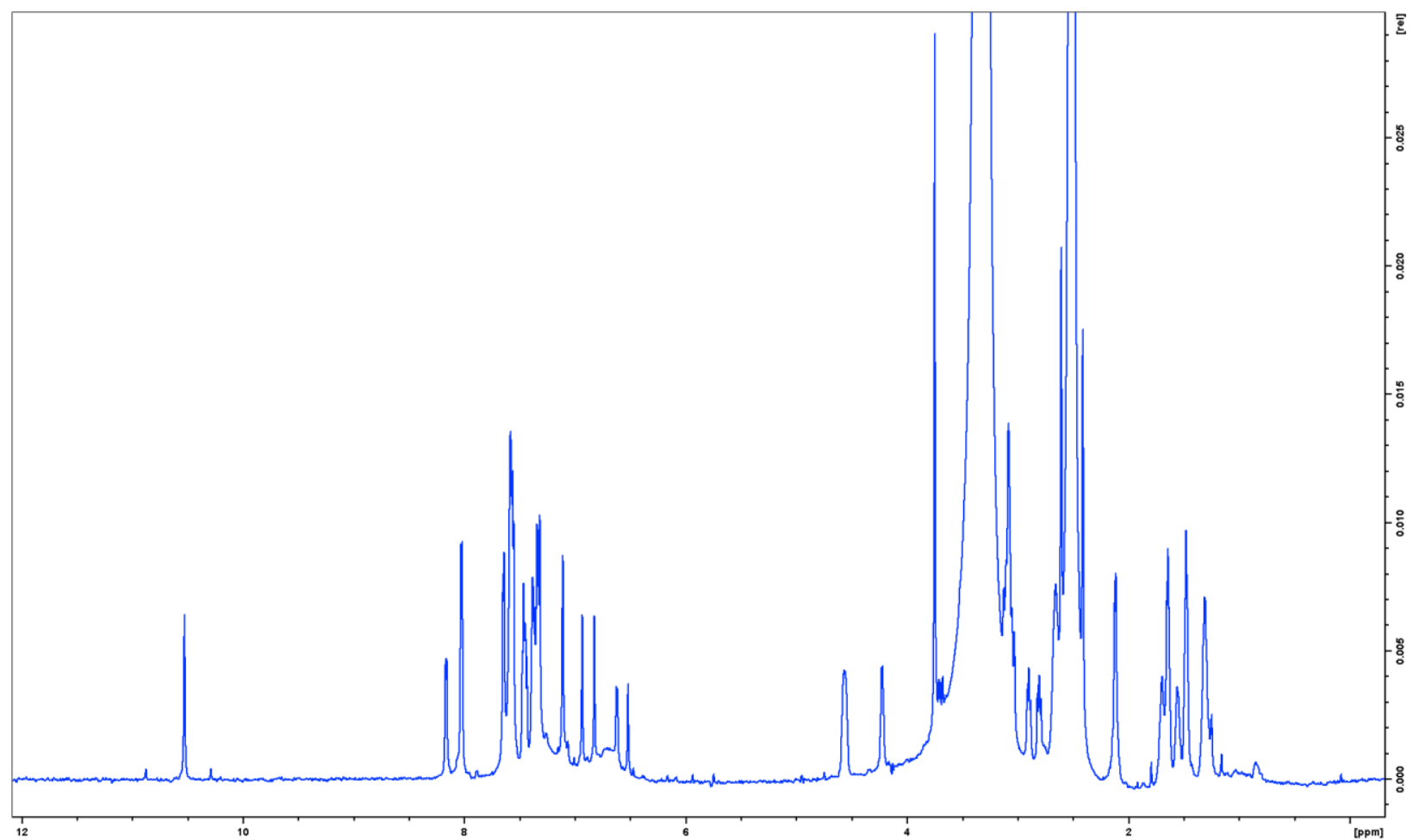

\section{Compound 11}

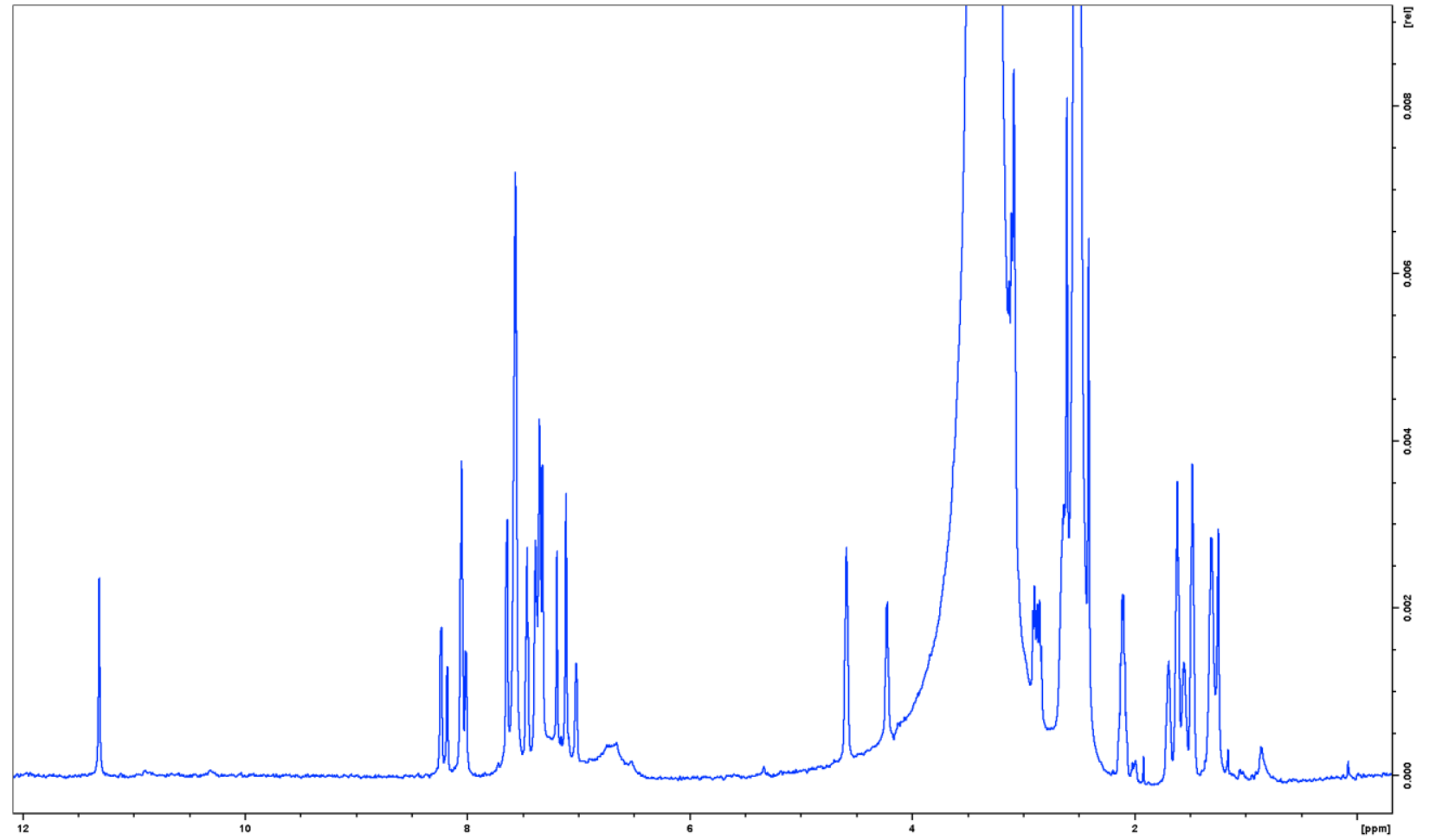


Figure S6. General synthetic scheme, high-resolution mass, and 1D ${ }^{1} \mathrm{H}$ NMR spectra for compounds in Table 3. Conditions: (a) 20\% 4-methylpiperidine in DMF, rt. (b) Fmoc-L-HomoArg(Pbf)-OH (3 eq.), HATU (3 eq.), Oxyma Pure (3 eq.), DIPEA (5 eq.), 1 h, rt. (c) R: Fmoc-3-(1-naphthyl)-L-alanine (3 eq.) for compound 12, Fmoc-3-(2-naphthyl)-L-alanine (3 eq.) for compound 13, Fmoc-4-(4-Chlorophenyl)-Lphenylalanine (3 eq.) for compound 14, Fmoc-4-(4-Methylphenyl)-L-phenylalanine (3 eq.) for compound 15, Fmoc-4-(2-Methylphenyl)-L-phenylalanine (3 eq.) for compound 16, Fmoc-4-(2-Methoxyphenyl)-Lphenylalanine (3 eq.) for compound 17, and Fmoc-4-(2,6-Dimethoxyphenyl)-L-phenylalanine (3 eq.) for compound 18, HATU (3 eq.), Oxyma Pure (3 eq.), DIPEA (5 eq.), 1 h, rt. (d) Fmoc-5-Hydroxy-Ltryptophan (3 eq.), HATU (3 eq.), Oxyma Pure (3 eq.), DIPEA (5 eq.), 1 h, rt. (e) $\gamma$-(Boc-amino)butyric acid (3 eq.), HATU (3 eq.), Oxyma Pure (3 eq.), DIPEA (5 eq.), 1 h, rt. (f) TFA/TIS/water/phenol (94:2:2:2), $5 \mathrm{~h}$, rt. High-resolution mass spectral data were acquired on an Agilent 6545 Q-TOF LC/MS instrument. NMR spectra were recorded on Bruker Avance III $700 \mathrm{MHz}$ equipped with a TCI cryoprobe.
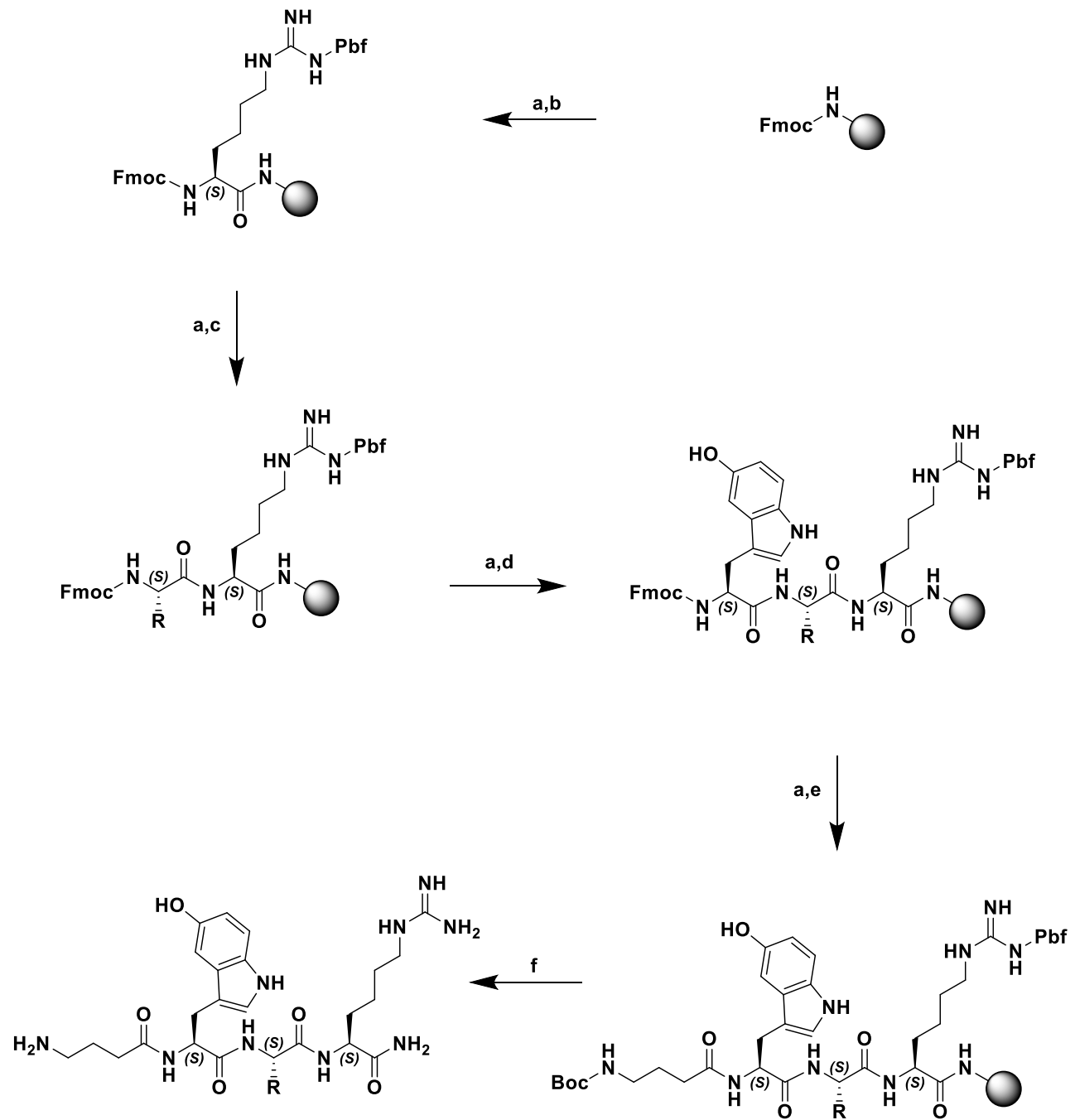


\begin{tabular}{|c|l|c|c|}
\hline $\mathbf{1}$ & \multicolumn{1}{|c|}{ IUPAC name } & $\begin{array}{c}\text { Calcd } \\
{[\mathbf{M + H}]^{+}}\end{array}$ & $\begin{array}{c}\text { Obs. } \\
{[\mathbf{M + H}]^{+}}\end{array}$ \\
\hline $\mathbf{1 2}$ & $\begin{array}{l}\text { (S)-2-((S)-2-((S)-2-(4-aminobutanamido)-3-(5-hydroxy-1H- } \\
\text { indol-3-yl)propanamido)-3-(naphthalen-1-yl)propanamido)-6- } \\
\text { guanidinohexanamide }\end{array}$ & 672.3617 & 672.3624 \\
\hline $\mathbf{1 3}$ & $\begin{array}{l}\text { (S)-2-((S)-2-((S)-2-(4-aminobutanamido)-3-(5-hydroxy-1H- } \\
\text { indol-3-yl)propanamido)-3-(naphthalen-2-yl)propanamido)-6- } \\
\text { guanidinohexanamide }\end{array}$ & 672.3617 & 672.3627 \\
\hline $\mathbf{1 4}$ & $\begin{array}{l}\text { (S)-2-((S)-2-((S)-2-(4-aminobutanamido)-3-(5-hydroxy-1H- } \\
\text { indol-3-yl)propanamido)-3-(4'-chloro-[1,1'-biphenyl]-4- } \\
\text { yl)propanamido)-6-guanidinohexanamide }\end{array}$ & 732.3383 & 732.3390 \\
\hline $\mathbf{1 5}$ & $\begin{array}{l}\text { (S)-2-((S)-2-((S)-2-(4-aminobutanamido)-3-(5-hydroxy-1H- } \\
\text { indol-3-yl)propanamido)-3-(4'-methyl-[1,1'-biphenyl]-4- } \\
\text { yl)propanamido)-6-guanidinohexanamide }\end{array}$ & 712.3930 & 712.3943 \\
\hline $\mathbf{1 6}$ & $\begin{array}{l}\text { (S)-2-((S)-2-((S)-2-(4-aminobutanamido)-3-(5-hydroxy-1H- } \\
\text { indol-3-yl)propanamido)-3-(2'-methyl-[1,1'-biphenyl]-4- } \\
\text { yl)propanamido)-6-guanidinohexanamide }\end{array}$ & 712.3930 & 712.3945 \\
\hline $\mathbf{1 7}$ & $\begin{array}{l}\text { (S)-2-((S)-2-((S)-2-(4-aminobutanamido)-3-(5-hydroxy-1H- } \\
\text { indol-3-yl)propanamido)-3-(2'-methoxy-[1,1'-biphenyl]-4- } \\
\text { yl)propanamido)-6-guanidinohexanamide }\end{array}$ & 728.3879 & 728.3882 \\
\hline $\mathbf{1 8}$ & $\begin{array}{l}\text { (S)-2-((S)-2-((S)-2-(4-aminobutanamido)-3-(5-hydroxy-1H- } \\
\text { indol-3-yl)propanamido)-3-(2',6'-dimethoxy-[1,1'-biphenyl]-4- } \\
\text { yl)propanamido)-6-guanidinohexanamide }\end{array}$ & 758.3984 & 758.4000 \\
\hline
\end{tabular}

\section{Compound 12}

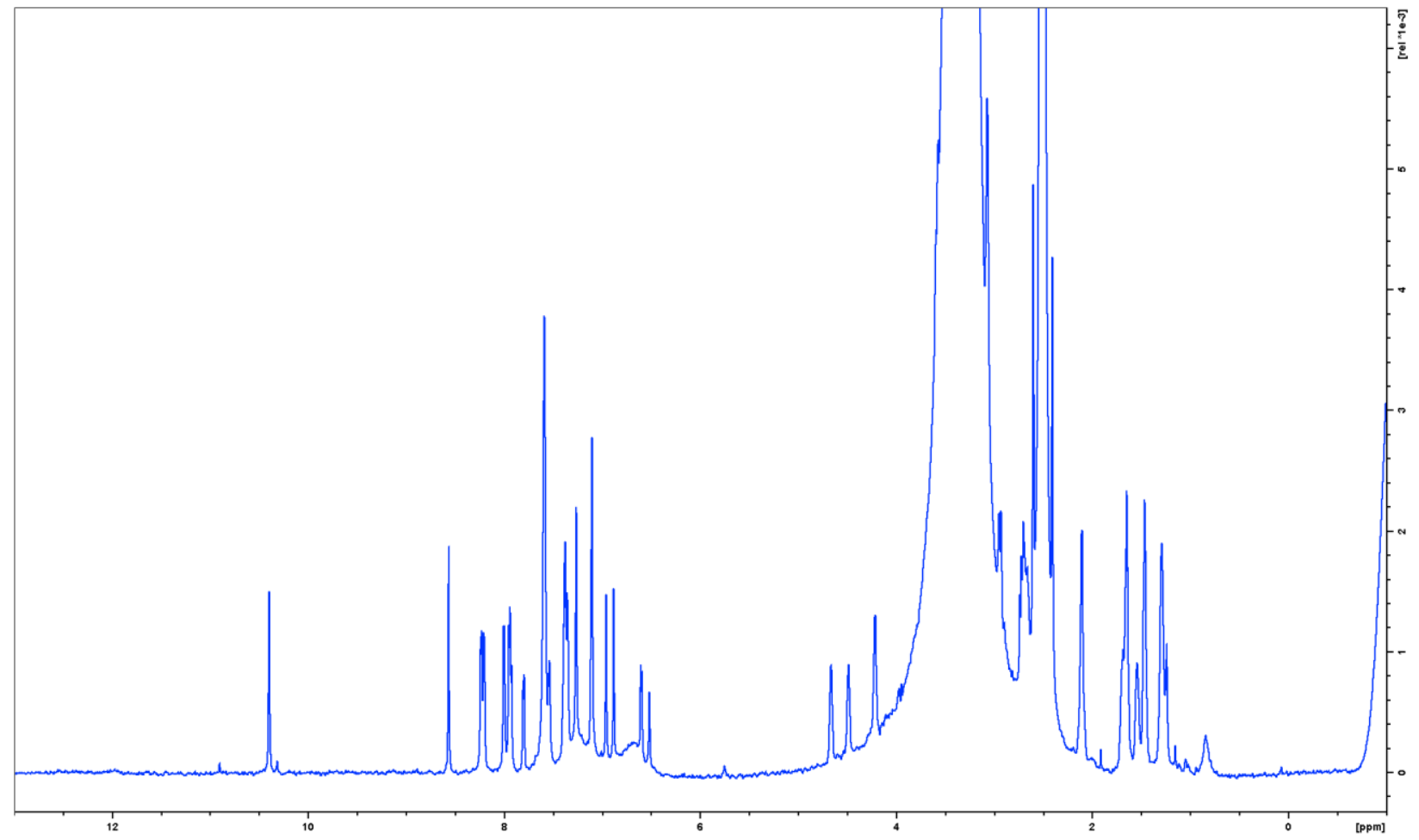




\section{Compound 13}

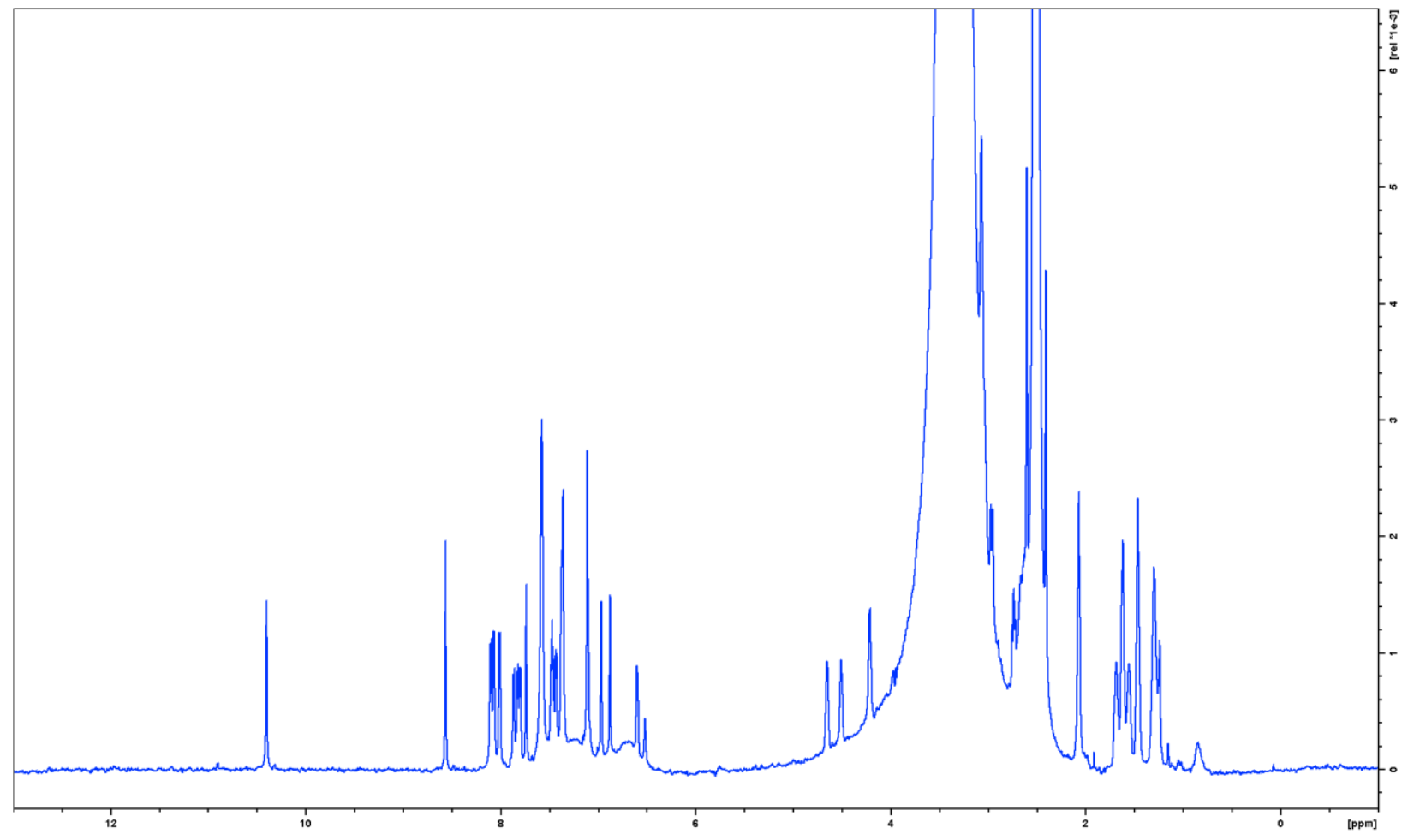

\section{Compound 14}

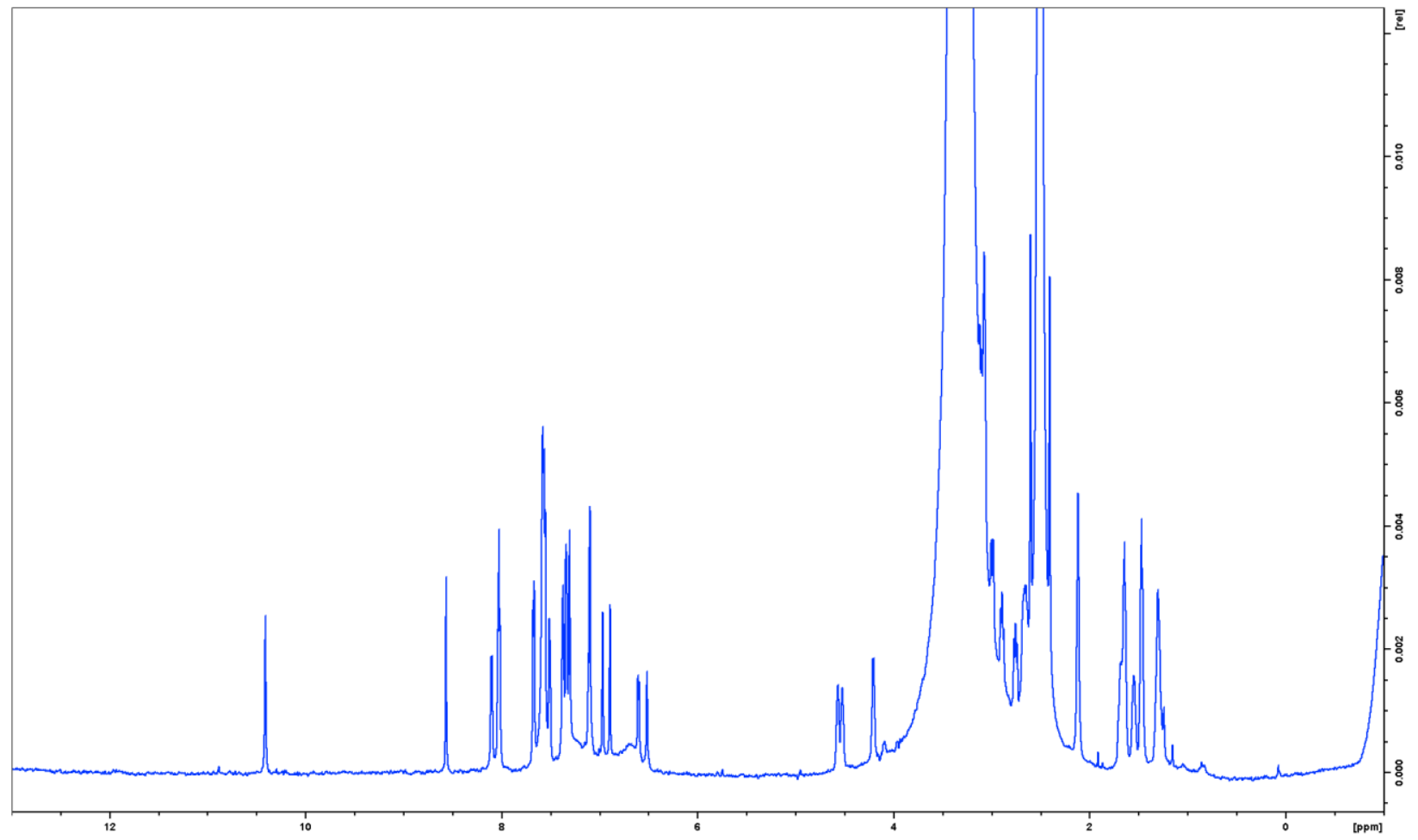




\section{Compound 15}

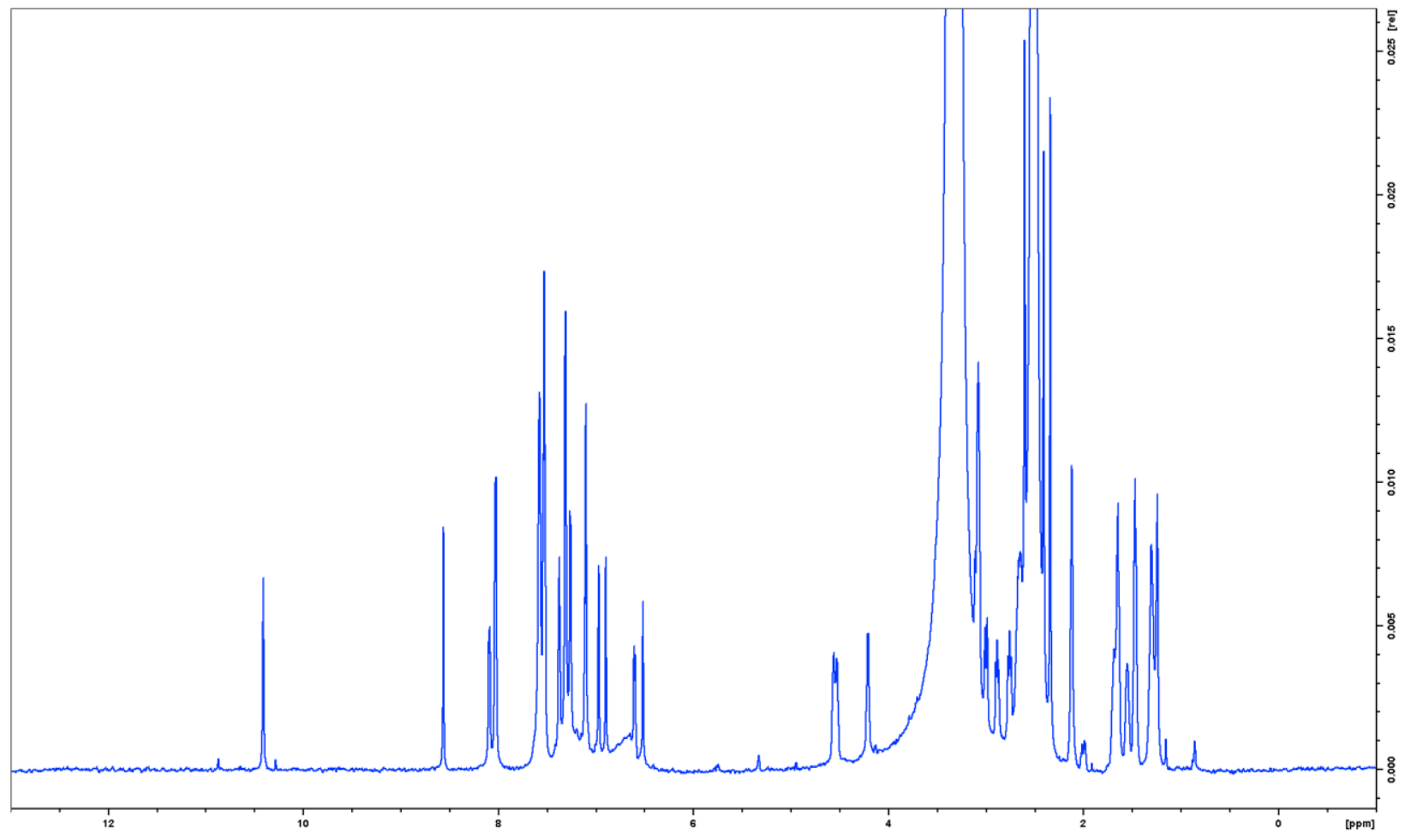

\section{Compound 16}

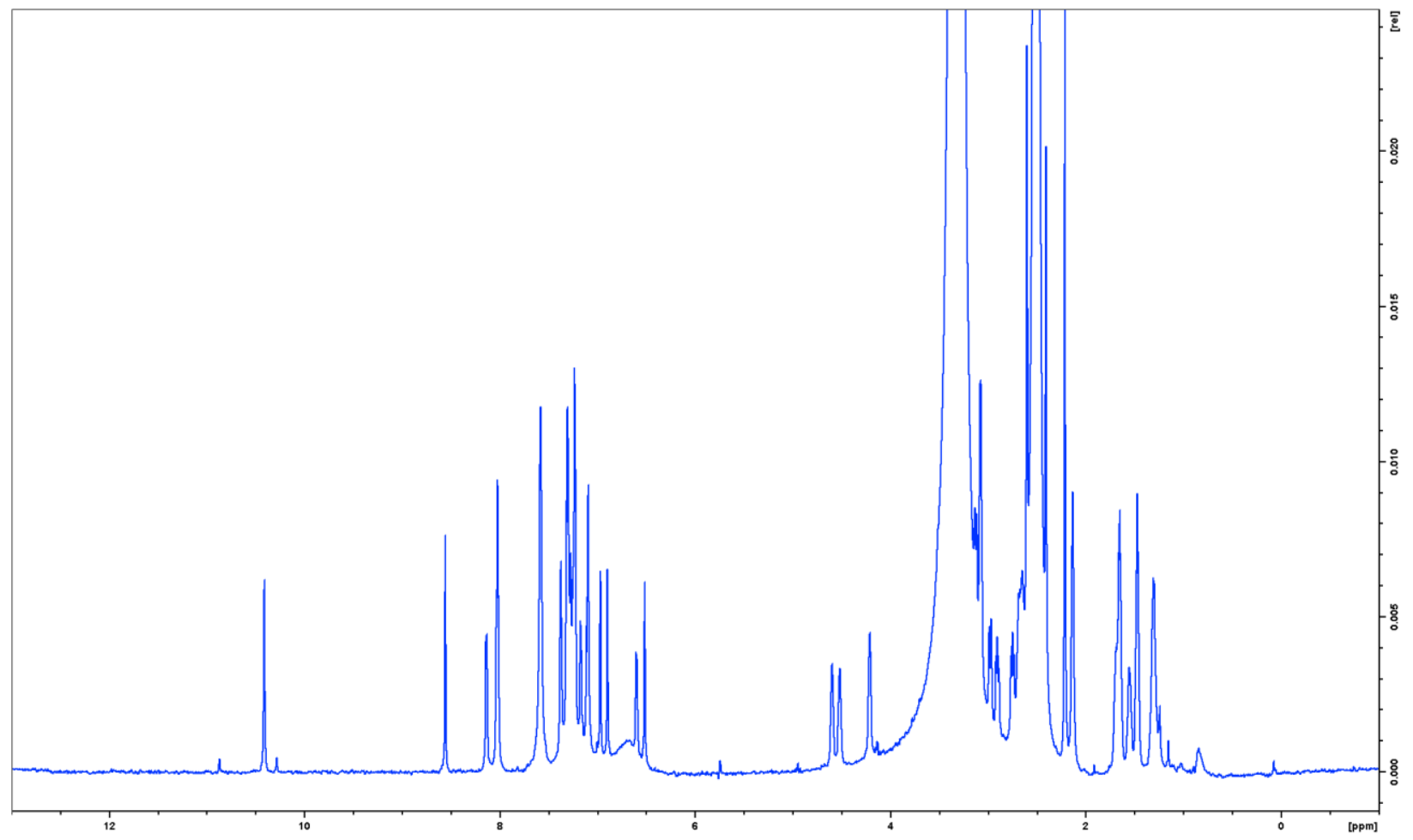




\section{Compound 17}

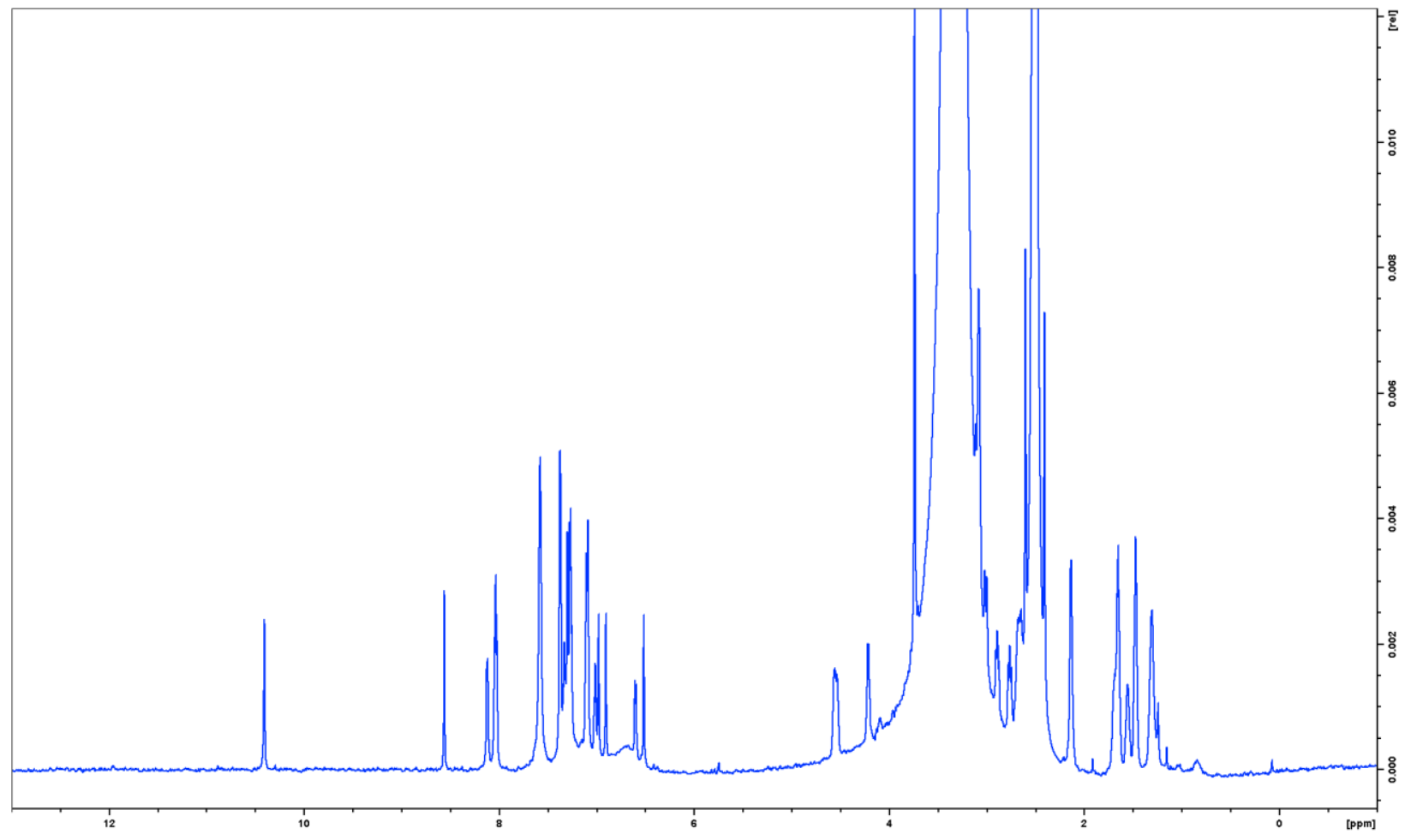

\section{Compound 18}

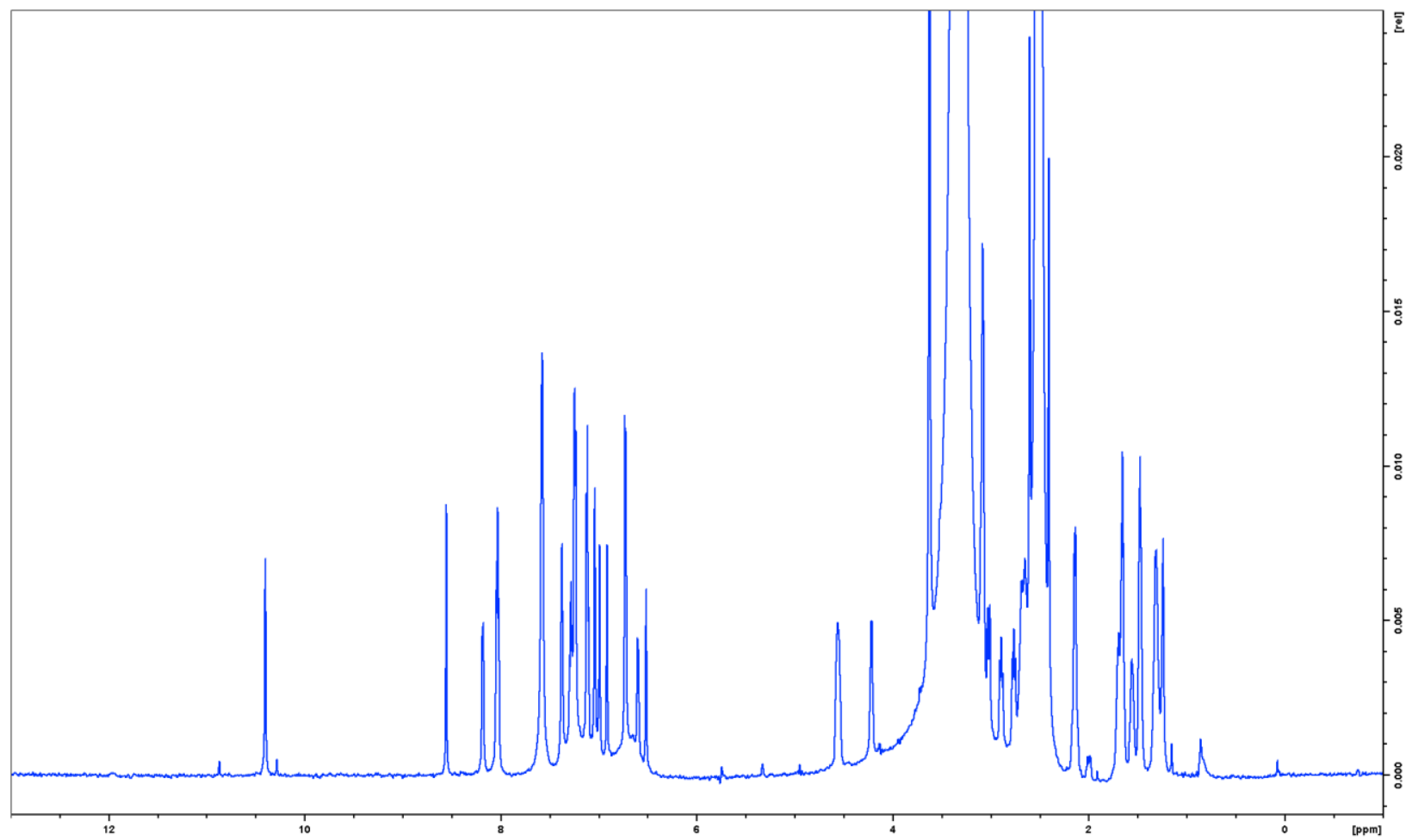


Figure S7. General synthetic scheme, high-resolution mass, and $1 \mathrm{D}^{1} \mathrm{H}$ NMR spectra for compounds in Table 4. Conditions: (a) 20\% 4-methylpiperidine in DMF, rt. (b) $\mathrm{R}_{1}$ : Fmoc-D-HomoArg(Pbf)-OH (3 eq.) for compound 20, Fmoc-L-Arg(Pbf)-OH (3 eq.) for compound 21, Fmoc-L-Lys(Boc)-OH (3 eq.) for compound 22, HATU (3 eq.), Oxyma Pure (3 eq.), DIPEA (5 eq.), 1 h, rt. (c) Fmoc-p-phenyl-Lphenylalanine ( 3 eq.), HATU (3 eq.), Oxyma Pure (3 eq.), DIPEA (5 eq.), 1 h, rt. (d) Fmoc-L-Trp(Boc) (3 eq.) for compound 20, Fmoc-5-Hydroxy-L-tryptophan (3 eq.) for compounds 21, and 22, HATU (3 eq.), Oxyma Pure (3 eq.), DIPEA (5 eq.), 1 h, rt. (e) $\gamma$-(Boc-amino)butyric acid (3 eq.), HATU (3 eq.), Oxyma Pure (3 eq.), DIPEA (5 eq.), 1 h, rt. (f) TFA/TIS/water/phenol (94:2:2:2), 5 h, rt. High-resolution mass spectral data were acquired on an Agilent 6545 Q-TOF LC/MS instrument. NMR spectra were recorded on Bruker Avance III $700 \mathrm{MHz}$ equipped with a TCI cryo-probe.
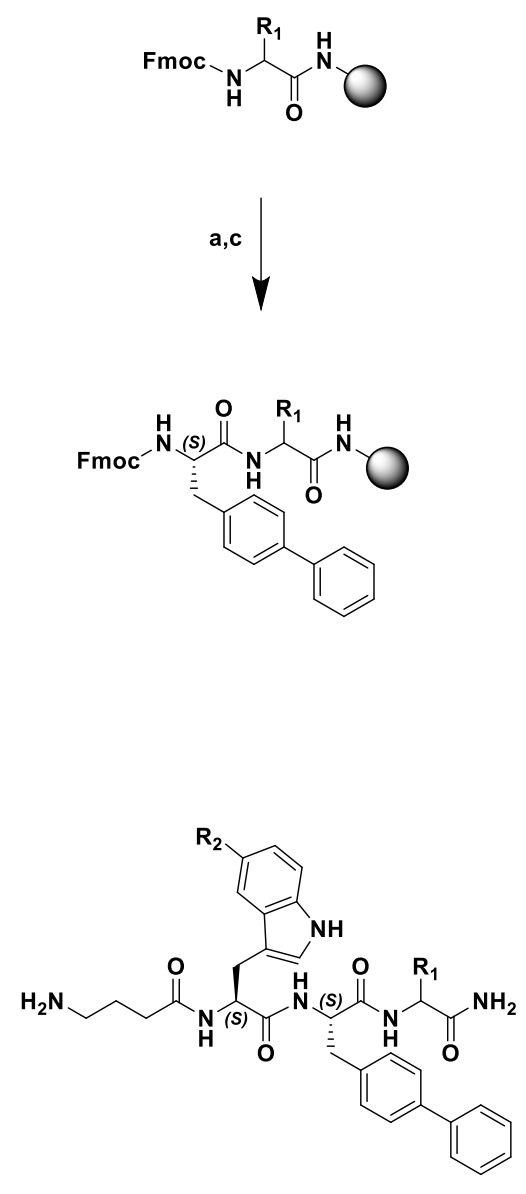

$R_{2}=H$ for 20

$\mathbf{R}_{\mathbf{2}}=\mathrm{OH}$ for 21,22
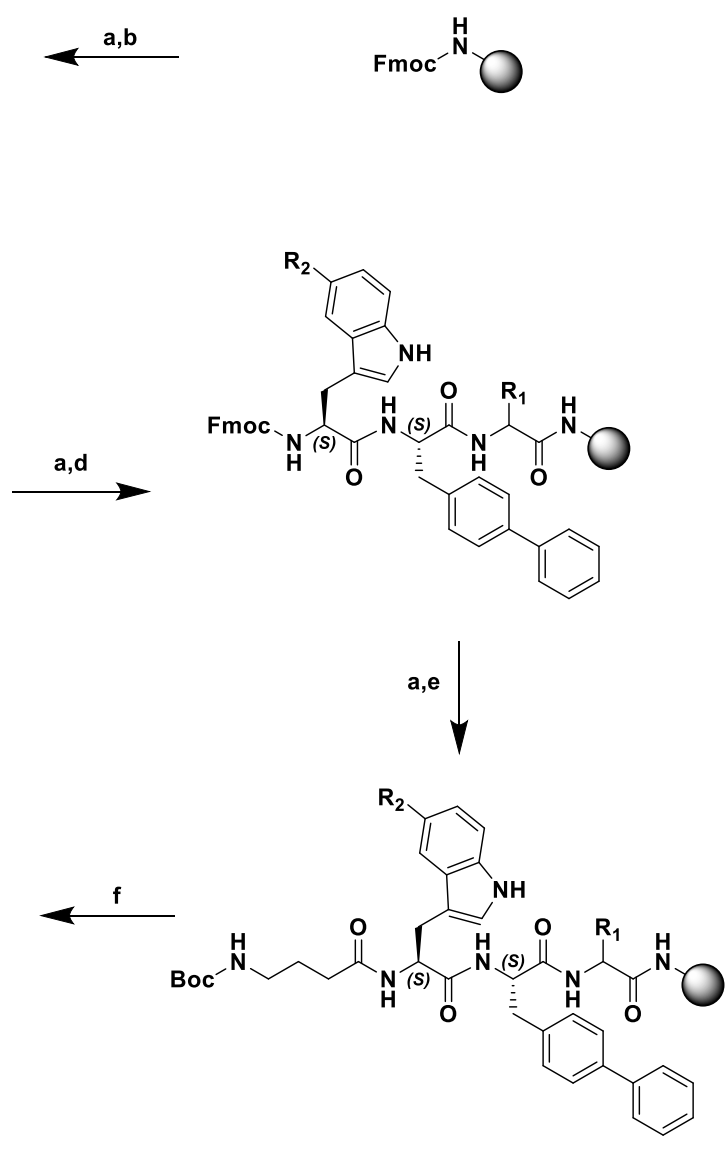


\begin{tabular}{|c|c|c|c|}
\hline ID & IUPAC name & $\begin{array}{c}\text { Calcd } \\
{[\mathrm{M}+\mathrm{H}]^{+}}\end{array}$ & $\begin{array}{c}\text { Obs. } \\
{[\mathrm{M}+\mathbf{H}]^{+}}\end{array}$ \\
\hline 19 & $\begin{array}{l}\mathrm{N}-((\mathrm{S})-1-(((\mathrm{S})-3-([1,1 \text { '-biphenyl]-4-yl)-1-(((S)-1-amino-3-(4- } \\
\text { guanidinophenyl)-1-oxopropan-2-yl)amino)-1-oxopropan-2- } \\
\text { yl)amino)-3-(1H-indol-3-yl)-1-oxopropan-2-yl)-4- } \\
\text { aminobutanamide }\end{array}$ & 716.3668 & 716.3673 \\
\hline 20 & $\begin{array}{l}\text { (R)-2-((S)-3-([1,1'-biphenyl]-4-yl)-2-((S)-2-(4- } \\
\text { aminobutanamido)-3-(1H-indol-3- } \\
\text { yl)propanamido)propanamido)-6-guanidinohexanamide }\end{array}$ & 682.3823 & 682.4343 \\
\hline 21 & $\begin{array}{l}\text { (S)-2-((S)-3-([1,1'-biphenyl]-4-yl)-2-((S)-2-(4- } \\
\text { aminobutanamido)-3-(5-hydroxy-1H-indol-3- } \\
\text { yl)propanamido)propanamido)-5-guanidinopentanamide }\end{array}$ & 684.3617 & 684.3634 \\
\hline 22 & $\begin{array}{l}\text { (S)-2-((S)-3-([1,1'-biphenyl]-4-yl)-2-((S)-2-(4- } \\
\text { aminobutanamido)-3-(5-hydroxy-1H-indol-3- } \\
\text { yl)propanamido)propanamido)-6-aminohexanamide }\end{array}$ & 656.3555 & 656.3568 \\
\hline
\end{tabular}

\section{Compound 19}

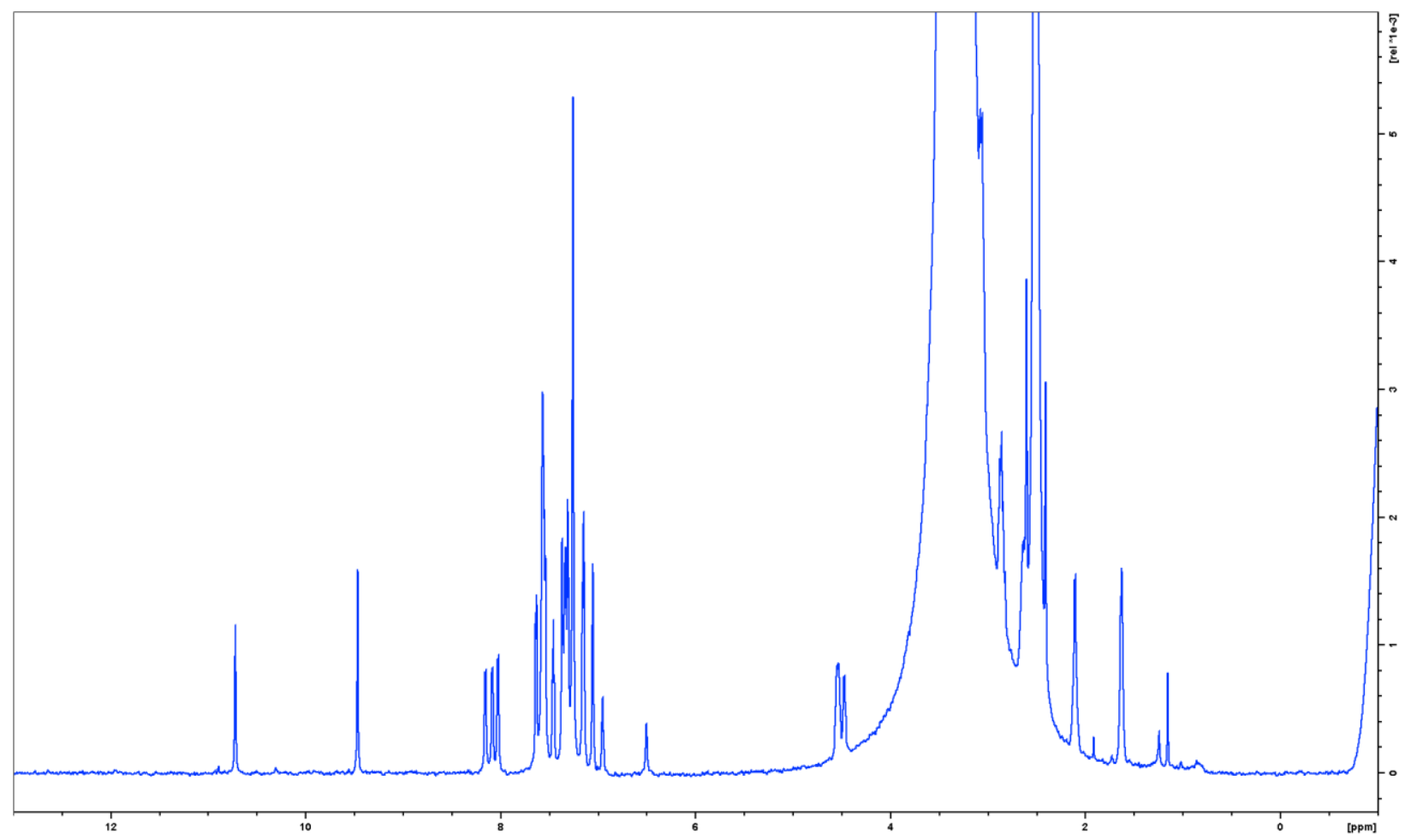




\section{Compound 20}

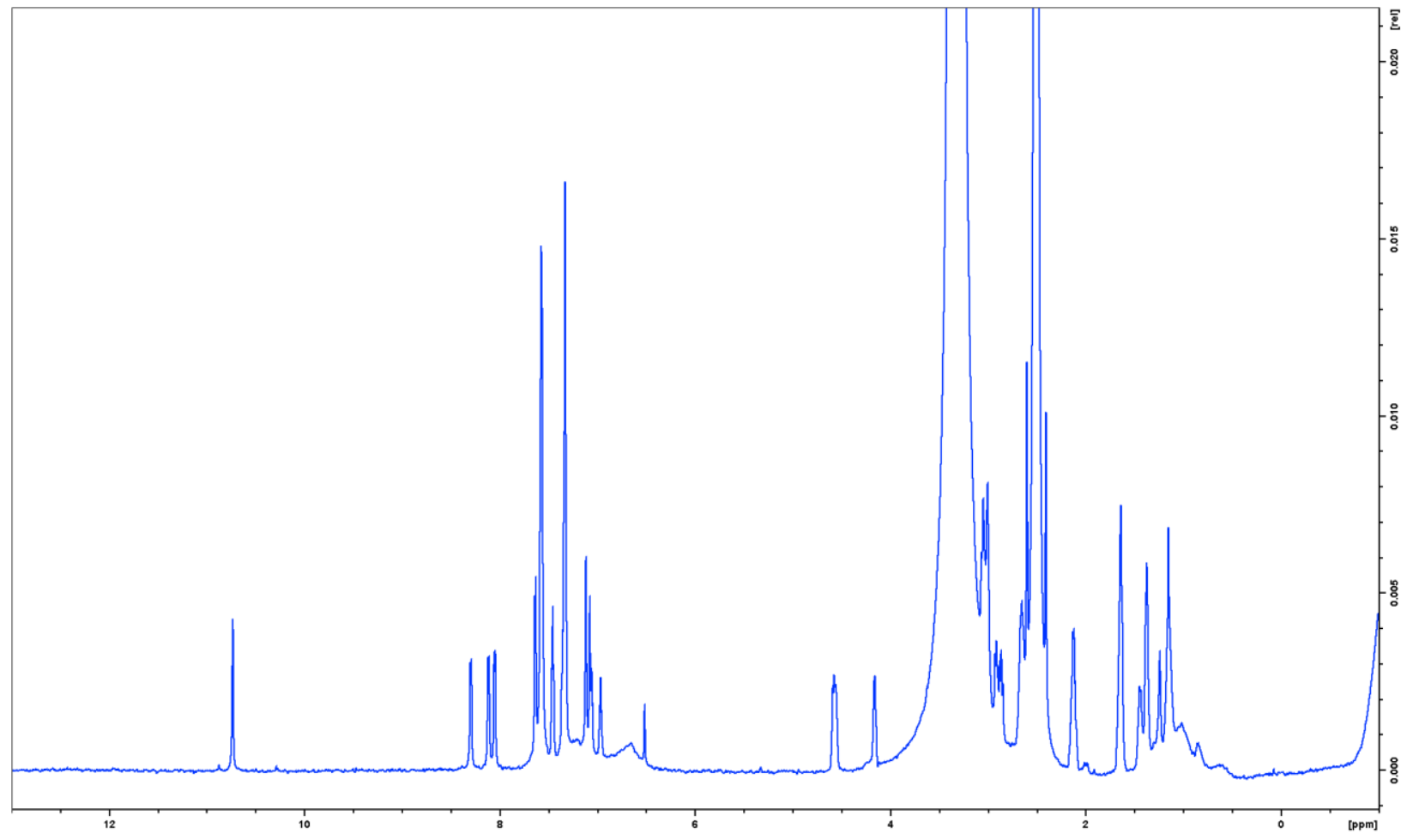

\section{Compound 21}

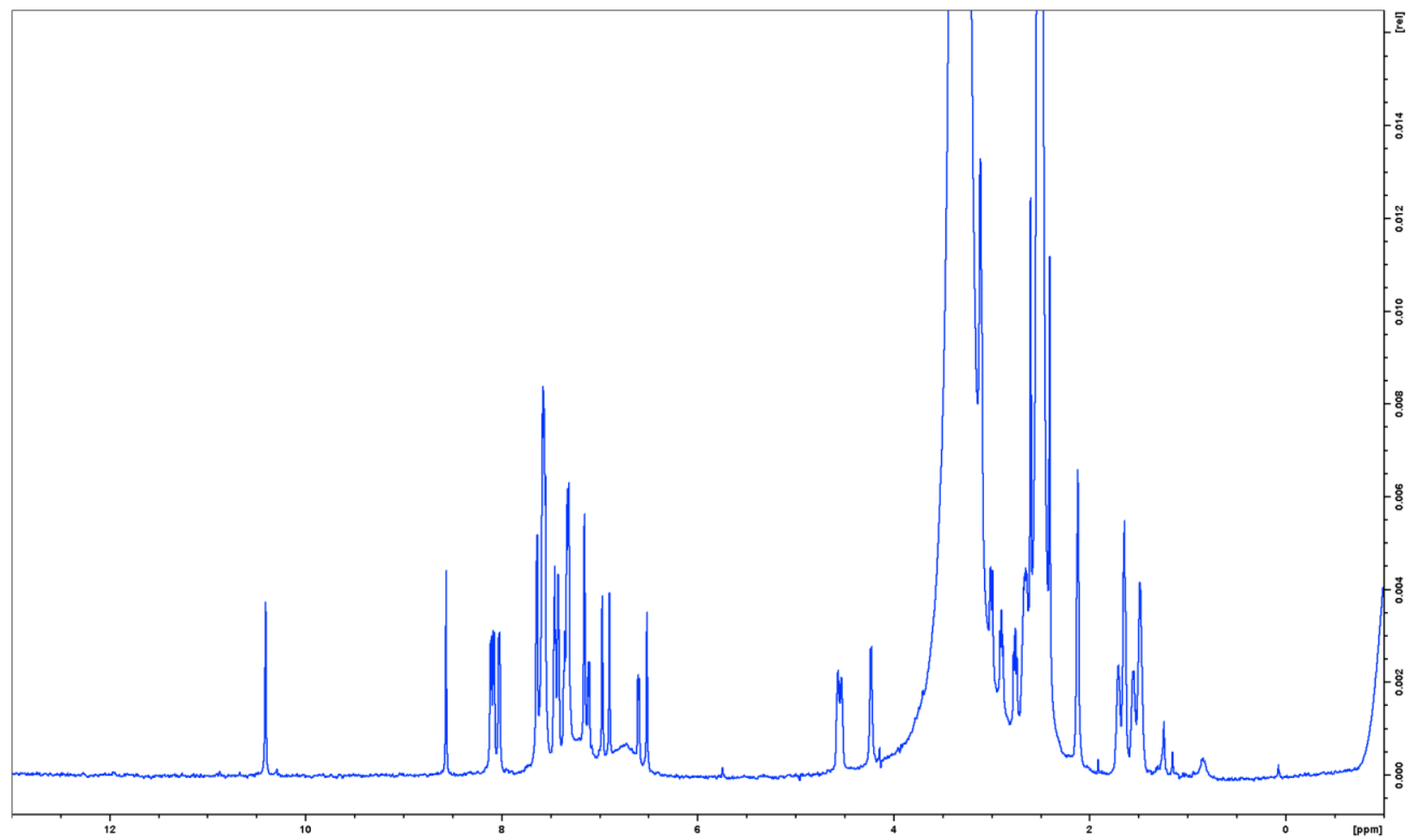




\section{Compound 22}

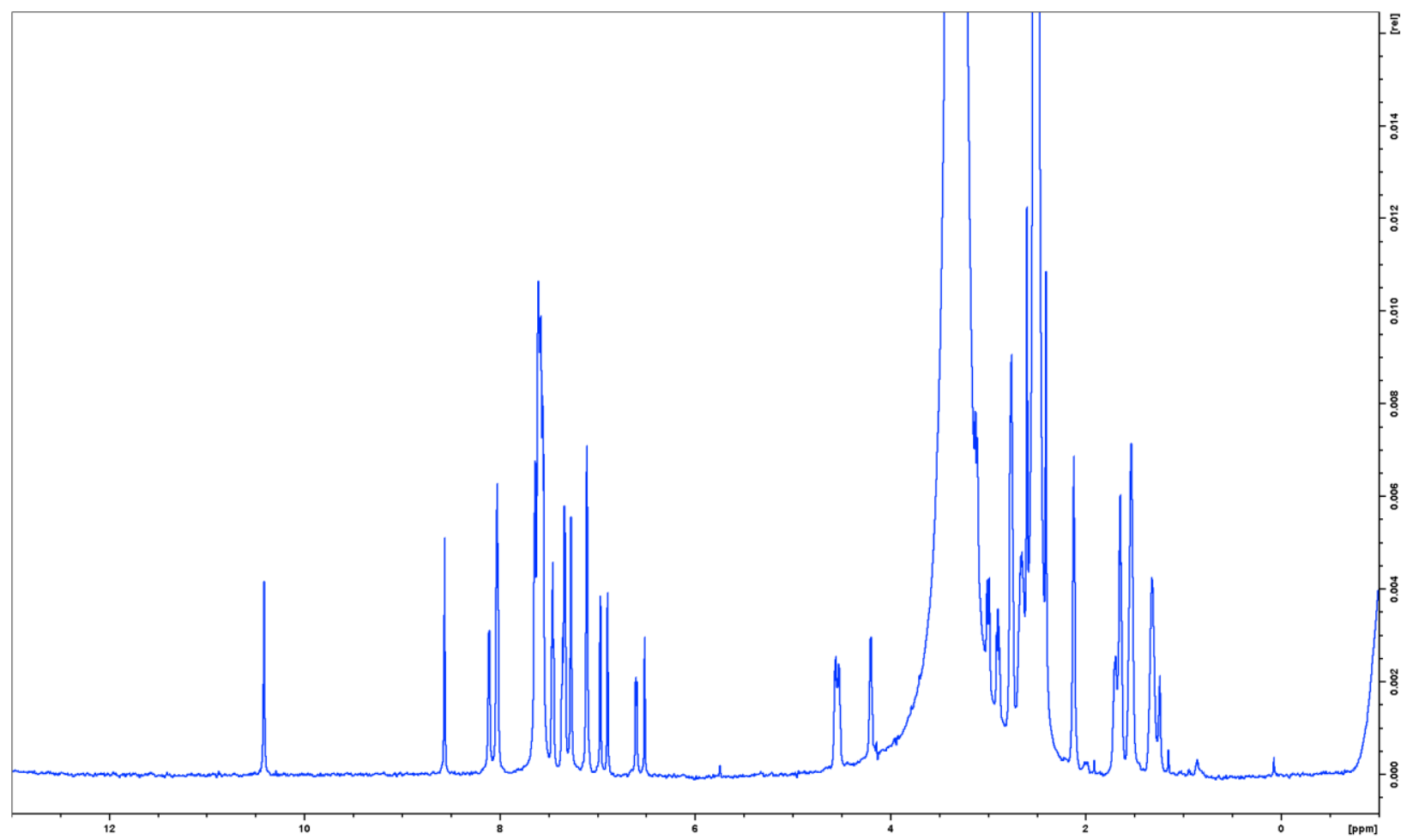


Figure S8. General synthetic scheme, high-resolution mass, and $1 \mathrm{D}^{1} \mathrm{H}$ NMR spectra for compounds in Table 5. Conditions: (a) R: 2-Amino-3-hydroxypyridine (3 eq.) for compound 23, Aniline (3 eq.) for compound 24, 3,5-Dimethoxybenzylamine (3 eq.) for compound 25, 2-Hydroxybenzylamine (3 eq.) for compound 26, 2-Aminophenol (3 eq.) for compound 27, 4-Morpholinoaniline (3 eq.) for compound 28, 4(Morpholinomethyl)aniline (3 eq.) for compound 29 in DMF, $30 \mathrm{~min}$, rt; (b) sodium triacetoxyborohydride (3 eq.), o/n, rt; (c) Fmoc-Gly-COOH (3 eq.), HATU (3 eq.), Oxyma Pure (3 eq.), DIPEA (5 eq.), 2 h, rt; (d) 20\% 4-methylpiperidine in DMF, rt; (e) Fmoc-L-HomoArg(Pbf)-OH (3 eq.), HATU (3 eq.), Oxyma Pure (3 eq.), DIPEA (5 eq.), 1 h, rt; (f) Fmoc-p-phenyl-L-phenylalanine (3 eq.), HATU (3 eq.), Oxyma Pure (3 eq.), DIPEA (5 eq.), 1 h, rt; (g) Fmoc-5-Hydroxy-L-tryptophan (3 eq.), HATU (3 eq.), Oxyma Pure (3 eq.), DIPEA (5 eq.), 1 h, rt; (h) $\gamma$-(Boc-amino)butyric acid ( 3 eq.), HATU (3 eq.), Oxyma Pure (3 eq.), DIPEA (5 eq.), 1 h, rt; (g) TFA/TIS/water/phenol (94:2:2:2), 5 h, rt. High-resolution mass spectral data were acquired on an Agilent 6545 Q-TOF LC/MS instrument. NMR spectra were recorded on Bruker Avance III $700 \mathrm{MHz}$ equipped with a TCI cryo-probe.

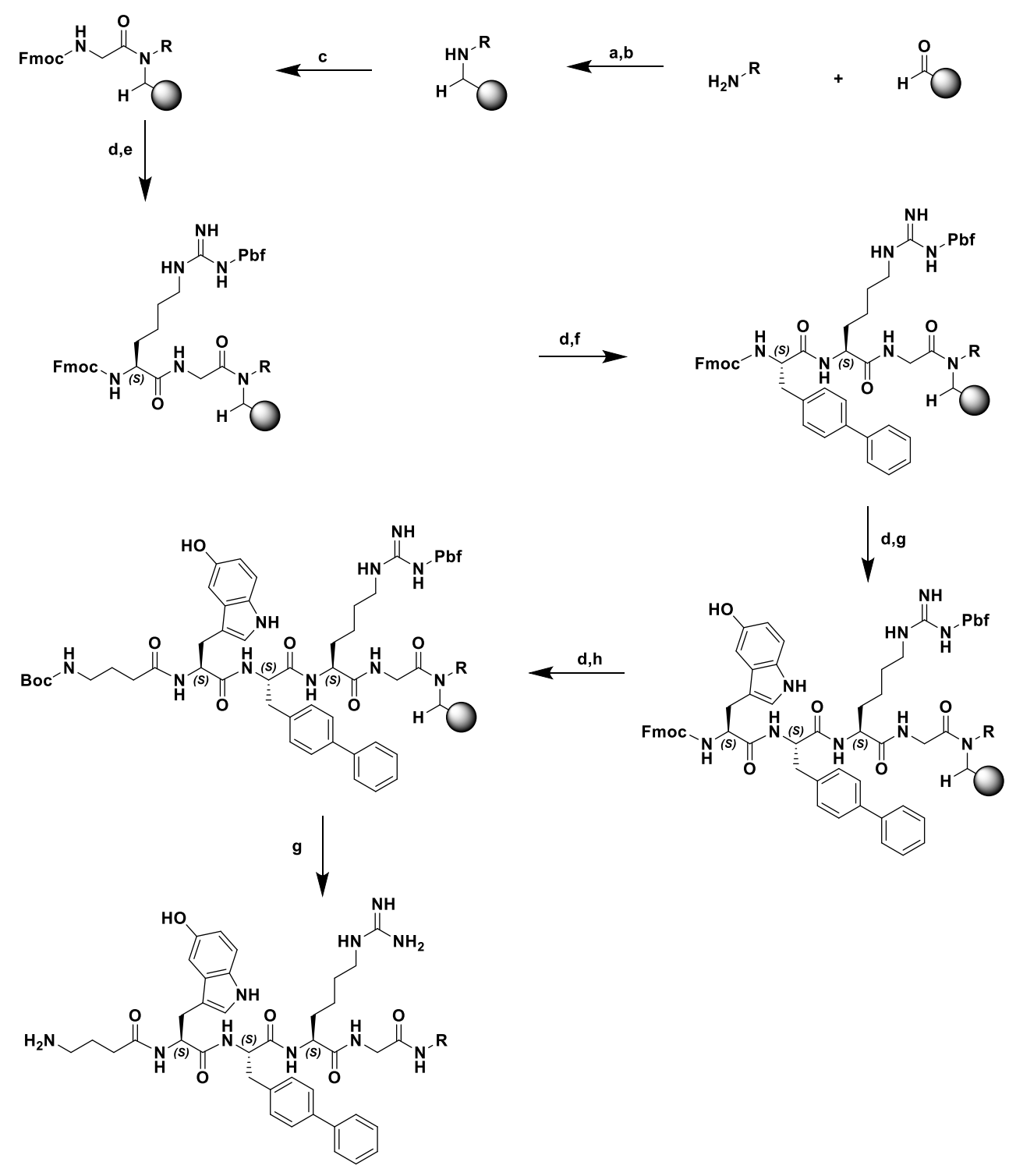




\begin{tabular}{|c|c|c|c|}
\hline ID & IUPAC name & $\begin{array}{c}\text { Calcd } \\
{[\mathrm{M}+\mathrm{H}]^{+}}\end{array}$ & $\begin{array}{c}\text { Obs. } \\
{[\mathbf{M}+\mathbf{H}]^{+}}\end{array}$ \\
\hline 23 & $\begin{array}{l}\text { (S)-2-((S)-3-([1,1'-biphenyl]-4-yl)-2-((S)-2-(4- } \\
\text { aminobutanamido)-3-(5-hydroxy-1H-indol-3- } \\
\text { yl)propanamido)propanamido)-6-guanidino-N-(2-((3- } \\
\text { hydroxypyridin-2-yl)amino)-2-oxoethyl)hexanamide }\end{array}$ & 848.4202 & 848.4221 \\
\hline 24 & $\begin{array}{l}\text { (S)-2-((S)-3-([1,1'-biphenyl]-4-yl)-2-((S)-2-(4- } \\
\text { aminobutanamido)-3-(5-hydroxy-1H-indol-3- } \\
\text { yl)propanamido)propanamido)-6-guanidino-N-(2-oxo-2- } \\
\text { (phenylamino)ethyl)hexanamide }\end{array}$ & 831.4300 & 831.4294 \\
\hline 25 & $\begin{array}{l}\text { (S)-2-((S)-3-([1,1'-biphenyl]-4-yl)-2-((S)-2-(4- } \\
\text { aminobutanamido)-3-(5-hydroxy-1H-indol-3- } \\
\text { yl)propanamido)propanamido)-N-(2-((3,5- } \\
\text { dimethoxybenzyl)amino)-2-oxoethyl)-6-guanidinohexanamide }\end{array}$ & 905.4669 & 905.4681 \\
\hline 26 & $\begin{array}{l}\text { (S)-2-((S)-3-([1,1'-biphenyl]-4-yl)-2-((S)-2-(4- } \\
\text { aminobutanamido)-3-(5-hydroxy-1H-indol-3- } \\
\text { yl)propanamido)propanamido)-6-guanidino-N-(2-((2- } \\
\text { hydroxybenzyl)amino)-2-oxoethyl)hexanamide }\end{array}$ & 861.4406 & 861.4414 \\
\hline 27 & $\begin{array}{l}\text { (S)-2-((S)-3-([1,1'-biphenyl]-4-yl)-2-((S)-2-(4- } \\
\text { aminobutanamido)-3-(5-hydroxy-1H-indol-3- } \\
\text { yl)propanamido)propanamido)-6-guanidino-N-(2-((2- } \\
\text { hydroxyphenyl)amino)-2-oxoethyl)hexanamide }\end{array}$ & 847.4250 & 847.4273 \\
\hline 28 & $\begin{array}{l}\text { (S)-2-((S)-3-([1,1'-biphenyl]-4-yl)-2-((S)-2-(4- } \\
\text { aminobutanamido)-3-(5-hydroxy-1H-indol-3- } \\
\text { yl)propanamido)propanamido)-6-guanidino-N-(2-((4- } \\
\text { morpholinophenyl)amino)-2-oxoethyl)hexanamide }\end{array}$ & 916.4828 & 916.4847 \\
\hline 29 & $\begin{array}{l}\text { (S)-2-((S)-3-([1,1'-biphenyl]-4-yl)-2-((S)-2-(4- } \\
\text { aminobutanamido)-3-(5-hydroxy-1H-indol-3- } \\
\text { yl)propanamido)propanamido)-6-guanidino-N-(2-((4- } \\
\text { (morpholinomethyl)phenyl)amino)-2-oxoethyl)hexanamide }\end{array}$ & 930.4985 & 930.4989 \\
\hline
\end{tabular}




\section{Compound 23}

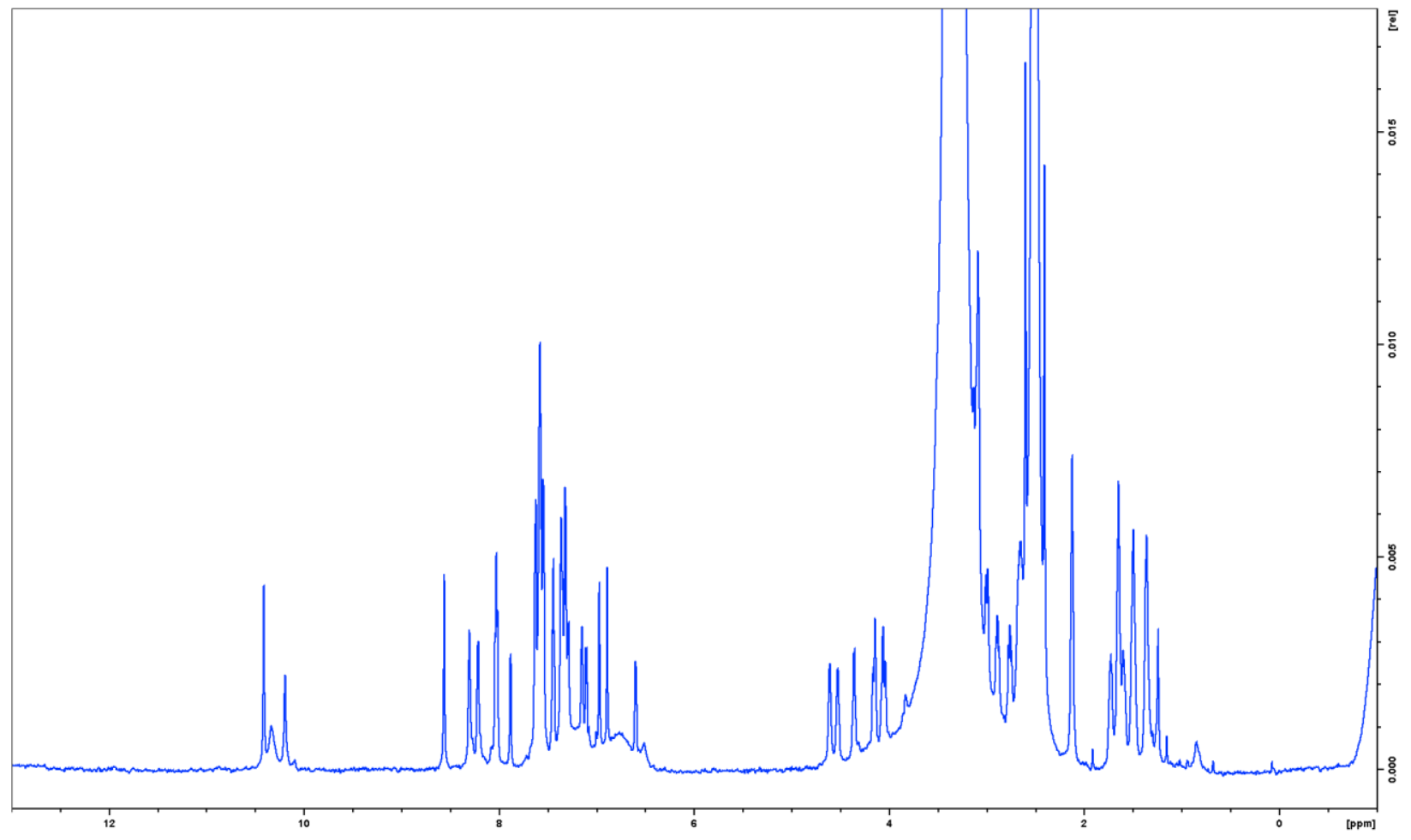

\section{Compound 24}

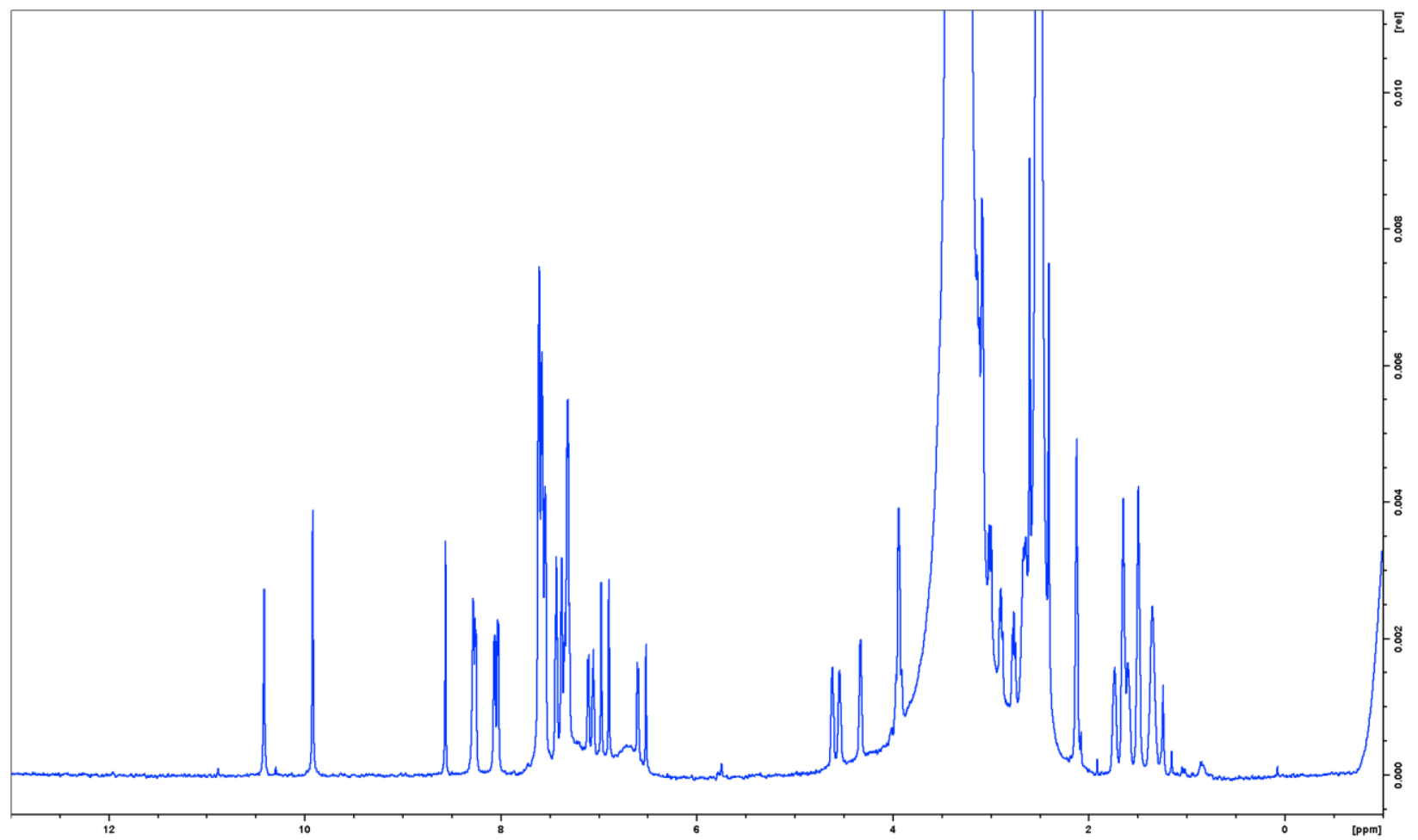




\section{Compound 25}

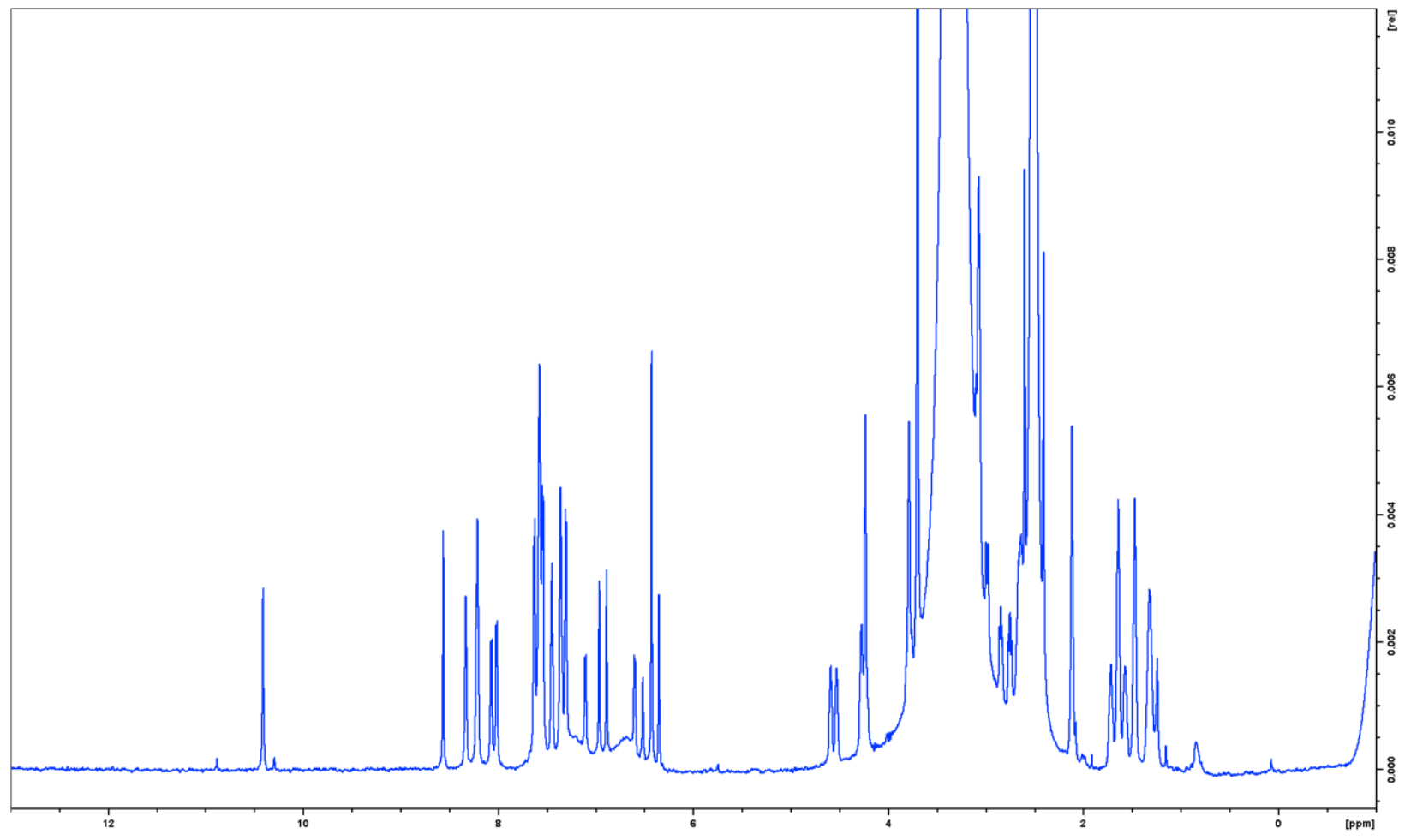

\section{Compound 26}

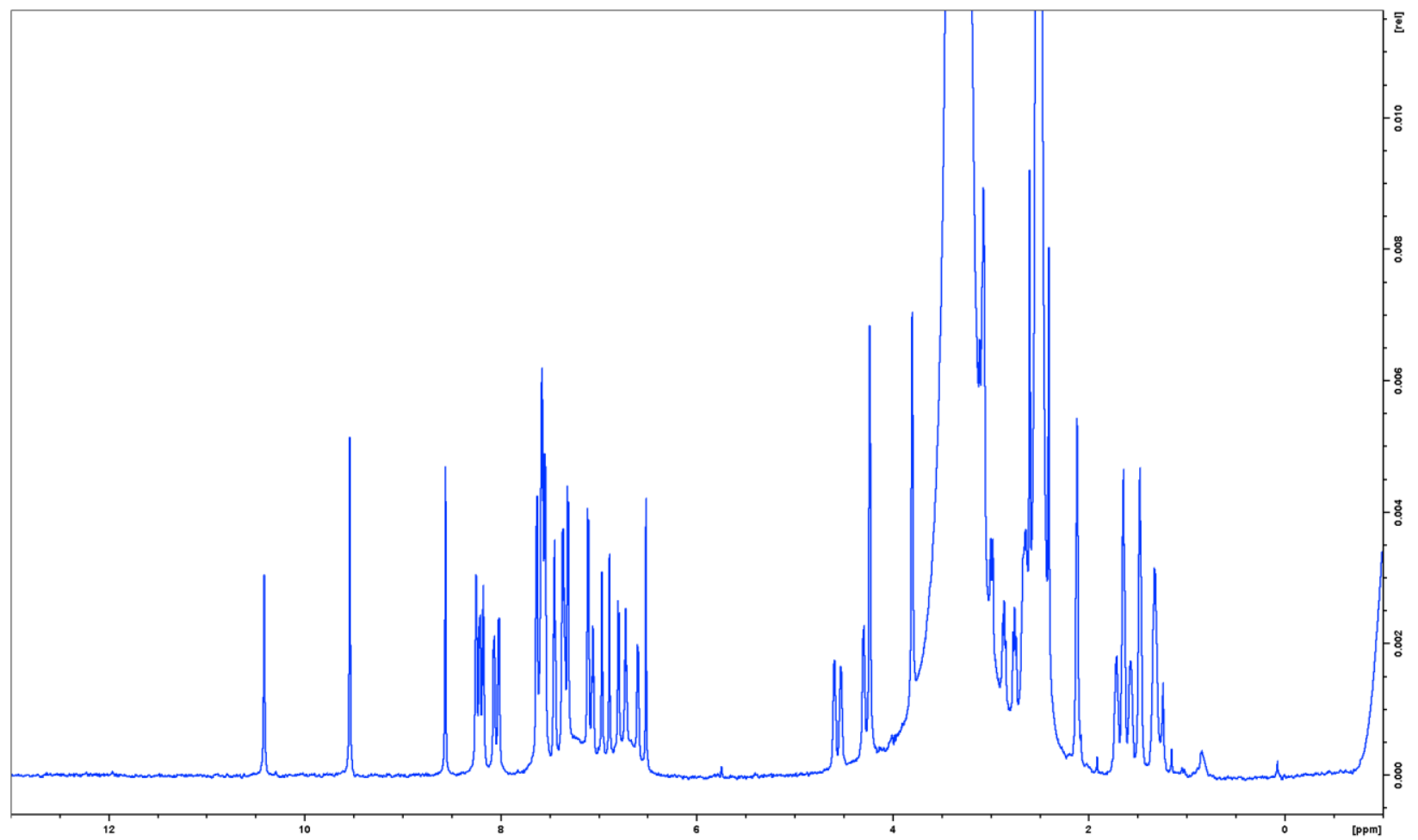




\section{Compound 27}

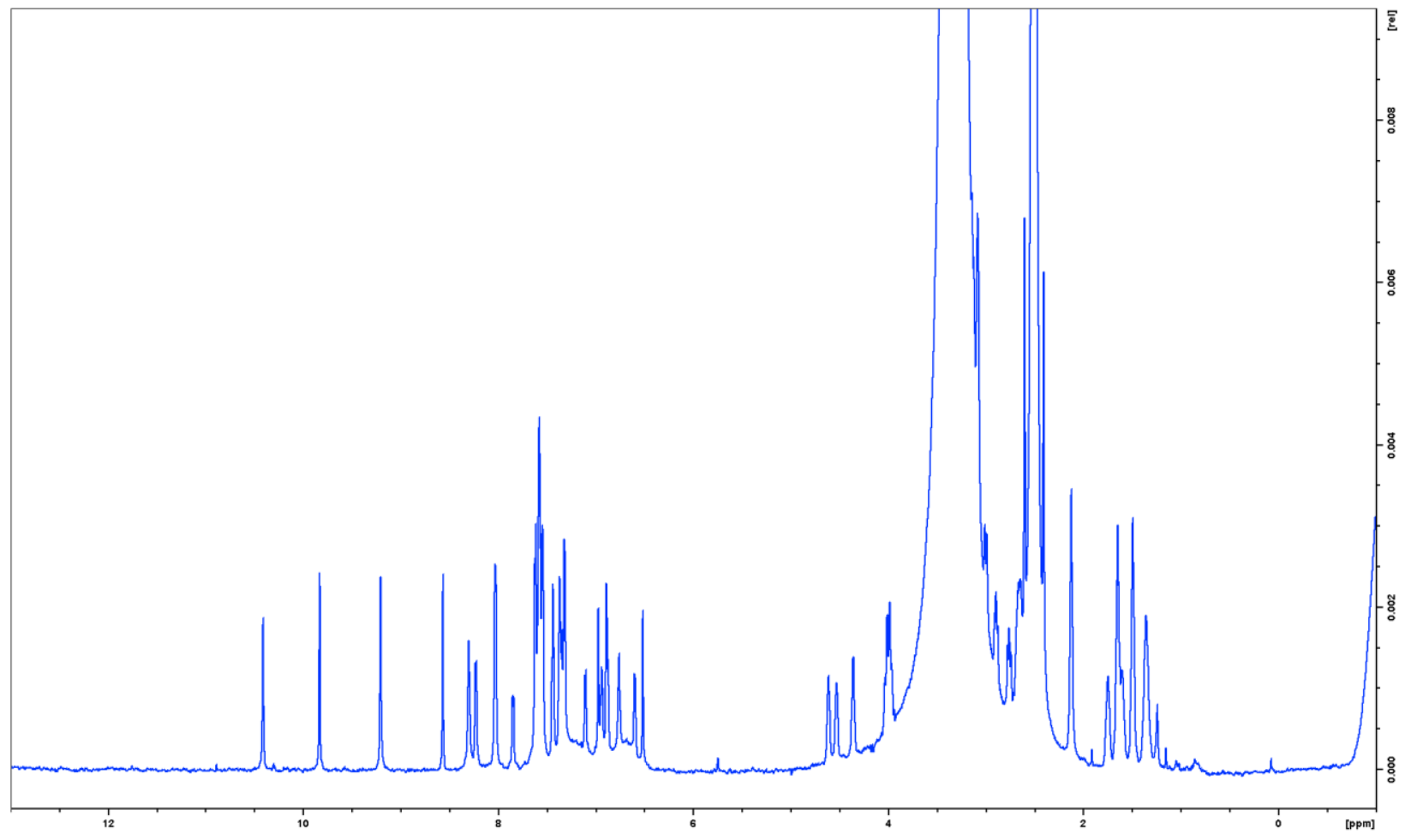

\section{Compound 28}

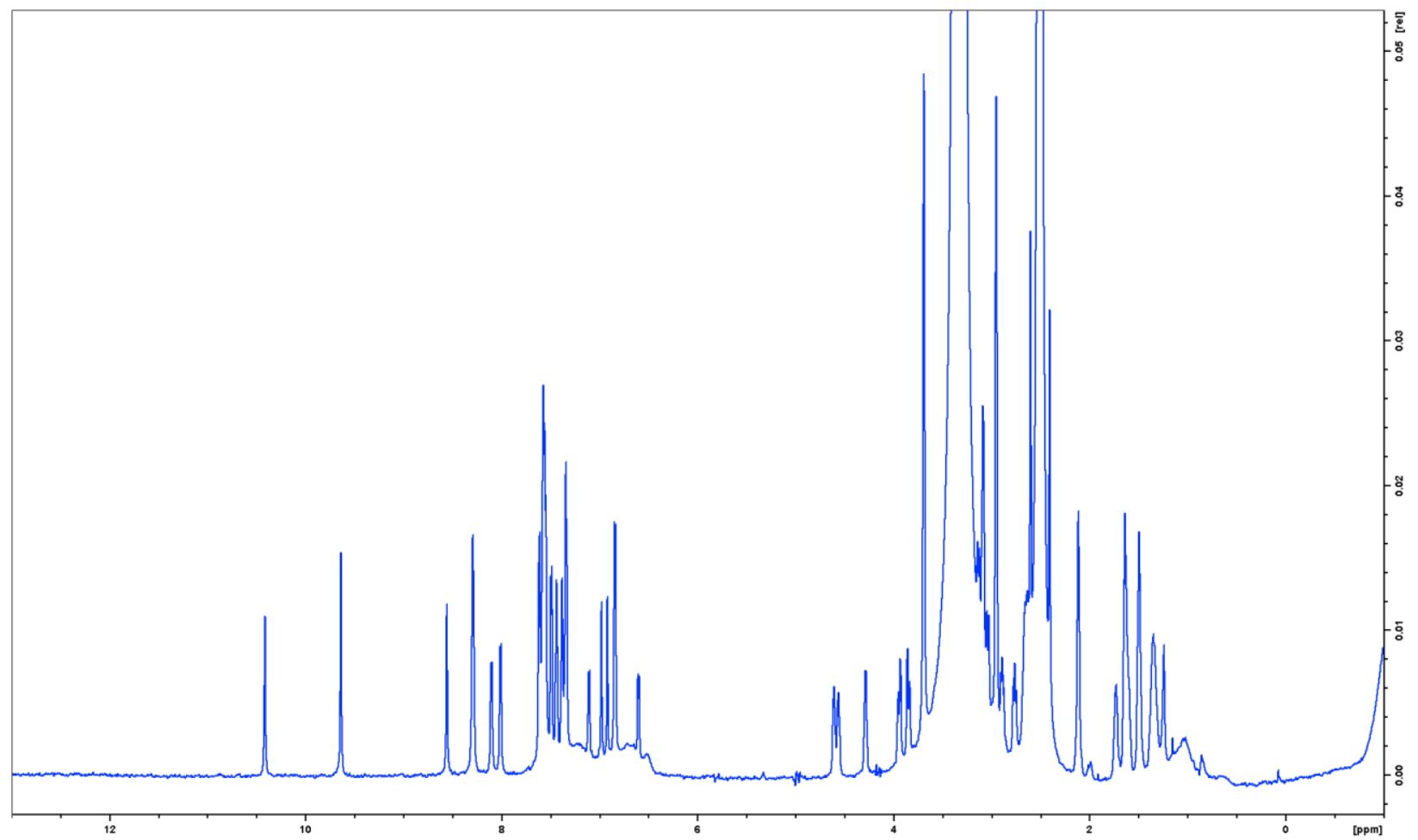




\section{Compound 29}

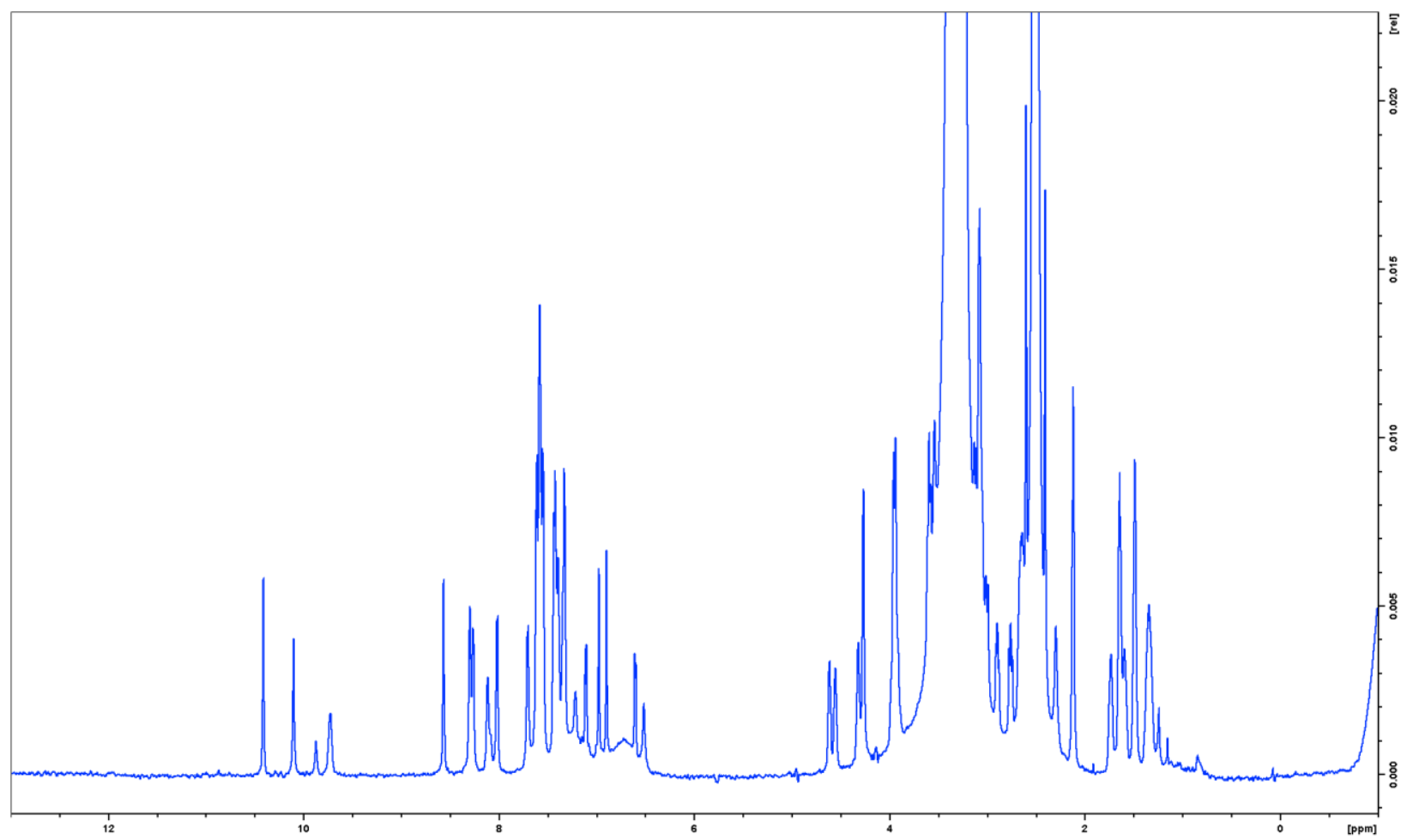


Figure S9. Original WB data that generated the data reported in Figure 5C,D.

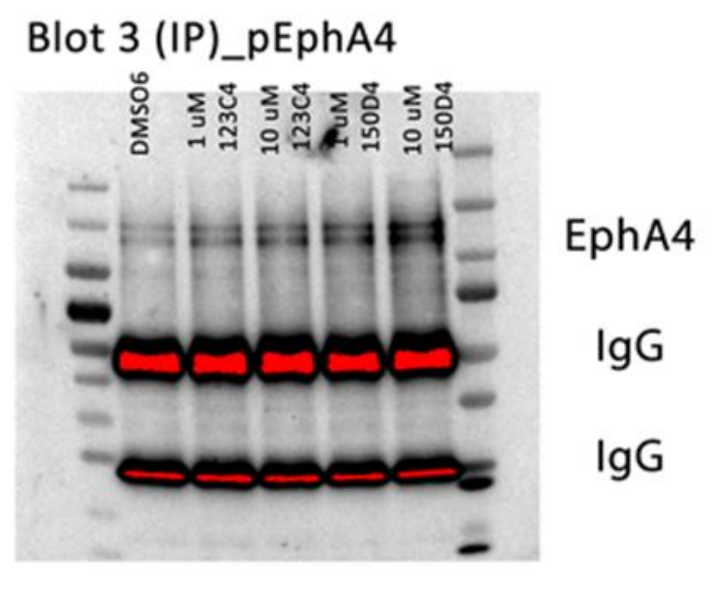

\section{Blot 3 (IP)_EphA4}

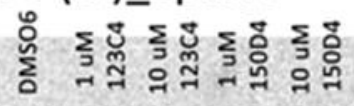

Blot 4 (IP)_pEphA4

Blot 4 (IP)_EphA4
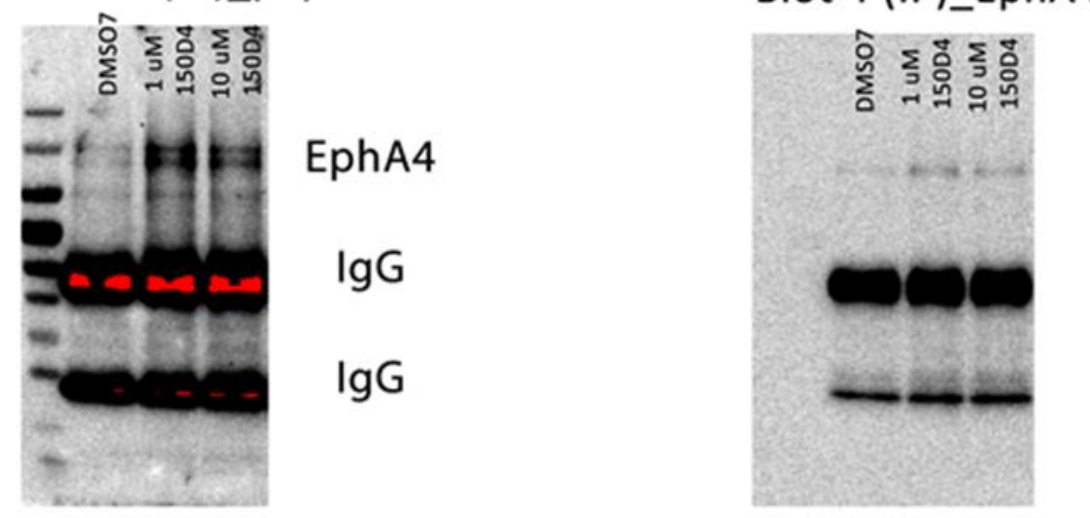

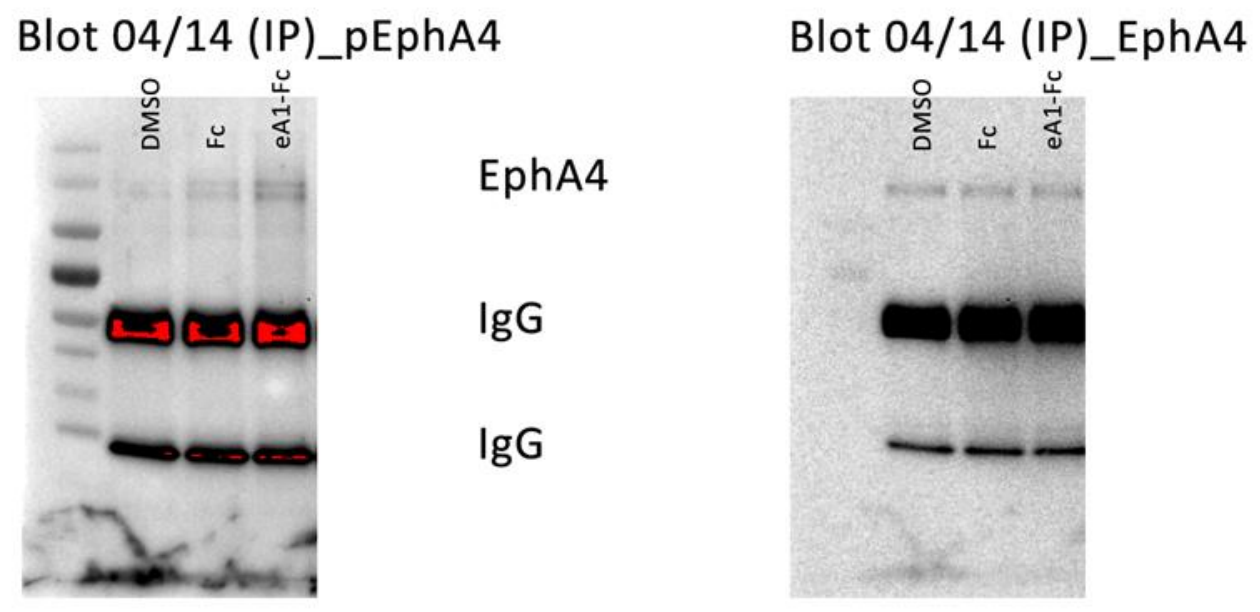

Blot 08/31 (IP)_pEphA4

商

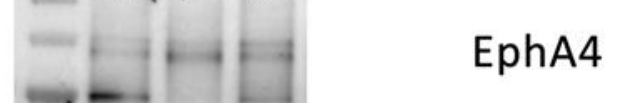

Blot 08/31 (IP)_EphA4
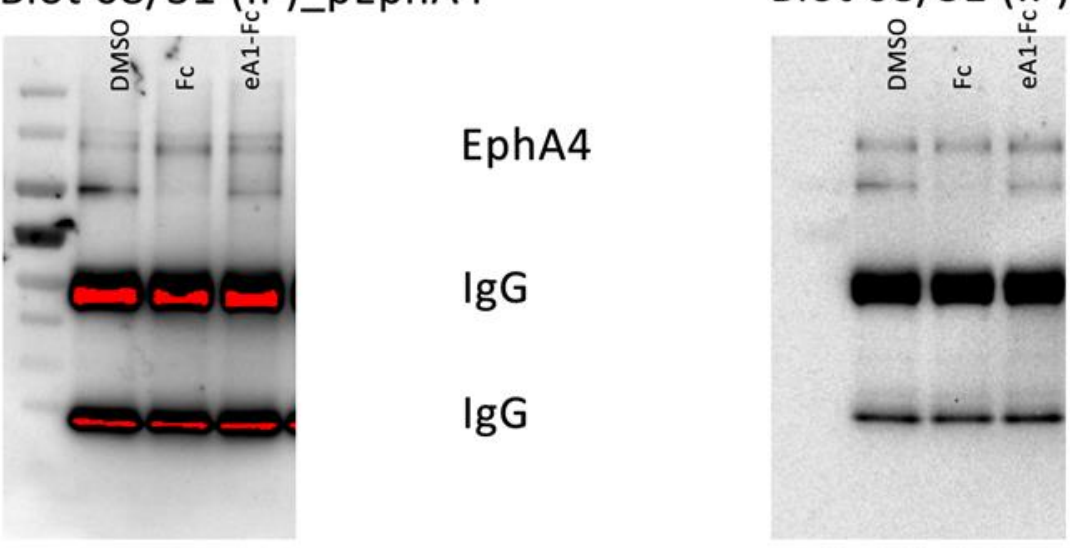

IgG

$\lg G$

Blot 09/01 (IP)_pEphA4

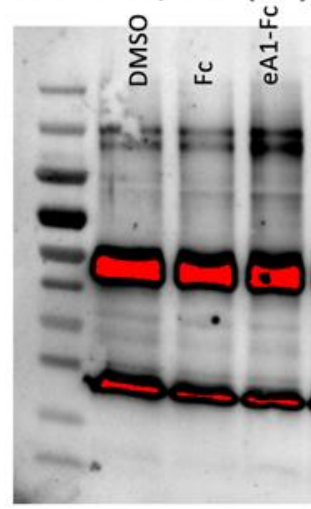

EphA4

$\lg G$

IgG

Blot 09/01 (IP)_EphA4

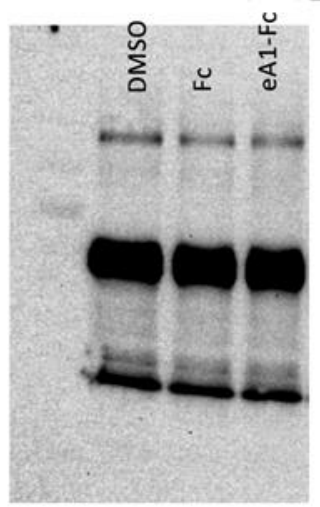

